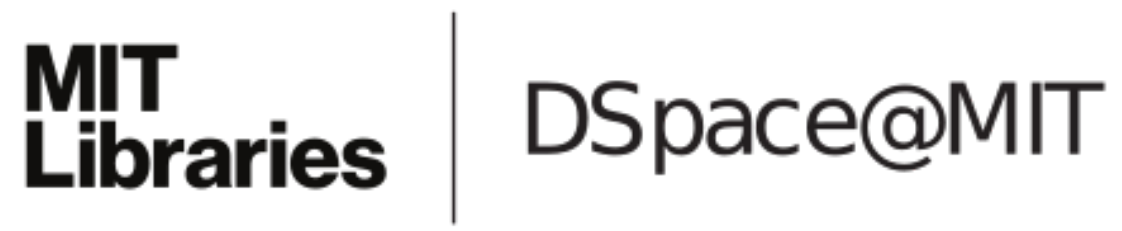

\author{
MIT Open Access Articles
}

Geometry mediated friction reduction in Taylor-Couette flow

The MIT Faculty has made this article openly available. Please share how this access benefits you. Your story matters.

Citation: Raayai-Ardakani, Shabnam and McKinley, Gareth H. 2020. "Geometry mediated friction reduction in Taylor-Couette flow." Physical Review Fluids, 5 (12).

As Published: 10.1103/PHYSREVFLUIDS.5.124102

Publisher: American Physical Society (APS)

Persistent URL: https://hdl.handle.net/1721.1/138878

Version: Final published version: final published article, as it appeared in a journal, conference proceedings, or other formally published context

Terms of Use: Article is made available in accordance with the publisher's policy and may be subject to US copyright law. Please refer to the publisher's site for terms of use. 


\title{
Geometry mediated friction reduction in Taylor-Couette flow
}

\author{
Shabnam Raayai-Ardakani $\odot^{*}$ and Gareth H. McKinley $\odot^{\dagger}$ \\ Department of Mechanical Engineering, Massachusetts Institute of Technology, \\ Cambridge, Massachusetts 02139, USA
}

(Received 26 March 2020; accepted 23 November 2020; published 28 December 2020)

\begin{abstract}
Periodic surface microtextures of different shapes such as $\mathrm{V}$ grooves, semicircular grooves, or rectangular grooves have been studied under laminar and turbulent flow conditions to offer guides for designing optimized low-friction surfaces. In this work we investigate the efficacy of periodic streamwise-aligned surface features in reducing the torque exerted on a steadily rotating cylinder in Taylor-Couette flow. Using threedimensional printed riblet-textured rotors and a bespoke Taylor-Couette cell, which can be mounted on a controlled stress rheometer, we measure the evolution in the torque acting on the inner rotor as a function of three different dimensionless parameters: (i) the Reynolds number characterizing the flow, (ii) the sharpness of the riblets, as defined by their aspect ratio (height to wavelength), and (iii) the axial scale of the riblets with respect to the size of the overall Taylor-Couette cell (the ratio of the riblet wavelength to the gap of the Taylor-Couette cell). Our experimental results in the laminar viscous flow regime show a reduction in torque up to $10 \%$ over a wide range of Reynolds numbers that is a nonmonotonic function of the aspect ratio of the grooves and independent of $\mathrm{Re}_{d}$ (the gap-based Reynolds number). However, after the transition to the Taylor vortex regime, the modification in torque also becomes a function of the Reynolds number while remaining a nonmonotonic function of the aspect ratio. Using finite-volume simulation of the three-dimensional swirling flow in the annular gap, we discuss the kinematic changes to the Taylor-Couette flow in the presence of the riblets compared to the case of smooth rotors and compute the resulting torque reduction as a function of the parameter space defined above. Good agreement between experiments and computational predictions is found for both azimuthal Couette flow and the Taylor vortex regime.
\end{abstract}

DOI: 10.1103/PhysRevFluids.5.124102

\section{INTRODUCTION}

Inspired by the riblets on the denticles of fast swimming shark species, drag reduction using riblet surfaces is one of several passive drag-reduction methods in which periodic microscale (or milliscale) grooves aligned in the flow direction are used to tune the net frictional drag force acting on the surfaces [1]. Experiments by Walsh and Lindemann have shown up to 8\% reduction in the viscous drag of flat plates in wind tunnel experiments. They used $\mathrm{V}$-groove and semicircular riblets to show that the drag reduction was a function of the shape of the riblets as well as the wavelength and amplitude of the riblets [1-3]. Hooshmand et al. also have reported a maximum of $7 \%$ in drag reduction in measurements of turbulent boundary layers over a V-groove riblet surface with a

\footnotetext{
*shraayai@mit.edu

† gareth@mit.edu
} 
height-to-spacing ratio of 0.5 [4]. Nakao has reported up to $8 \%$ reduction in the frictional resistance in pipe flow, using axially aligned $\mathrm{V}$-groove riblets with spacing of between 0.71 and $2.04 \mathrm{~mm}$ [5].

The effect of riblets on frictional drag reduction has also been considered in laminar flows [6-9]: Djenidi et al. showed 1-3\% reduction with $\vee$ grooves of spacing of $1 \mathrm{~mm}$ and heights of $0.4-2 \mathrm{~mm}$ in laminar boundary layers [7]. The primary mechanism of the reduction in laminar flow has been identified to be the flow retardation experienced inside the grooves that results in an increase in the thickness of the boundary layer [6-9]. This mechanism has also been observed in turbulent flows $[4,8,10,11]$. Using finite-volume simulations of the developing laminar boundary layer flow over sinusoidal riblets, we have recently shown that viscous drag reduction indeed depends on the local riblet geometry but also on the overall length of the riblet surface under study, because the kinematic features of the complex three-dimensional flow near the riblets evolve along the plate [12-14].

Fully developed Taylor-Couette flow (between two concentric cylinders), however, is a canonical internal flow which is not affected by entrance or exit effects. Consequently, the Taylor-Couette geometry [15-17] has been extensively used for exploring the levels of drag reduction that can be achieved using a number of different passive and active methods such as riblets $[18,19]$, bubbles [20,21], liquid infused surfaces [22], and textured superhydrophobic surfaces that support a stable plastron (air film) [23]. Hall and Joseph used riblets with a wavelength of $114 \mu \mathrm{m}$ and height (amplitude $A$ ) to wavelength $(\lambda)$ ratio of unity on the inner cylinder of a Taylor-Couette cell and were able to observe up to $5.1 \%$ reduction in drag [18]. Greidanus et al. [19] used a Taylor-Couette cell and a ribbed rotor with a wavelength of 0.012 times the gap between the cylinders and amplitude-to-wavelength ratio of 0.46 to study the reduction in the shear stress acting on the cylinder surface over a range of Reynolds numbers $10^{3}<\operatorname{Re}_{s}<10^{5}$, where the Reynolds number was defined as a function of the combination of the rotation rates of both the inner and outer cylinders. They were able to observe a reduction in the shear stress similar to the drag reduction reported previously by Bechert et al. [24] in a channel flow setting, as well as those reported by Hall and Joseph [18]. Zhu et al. also performed a set of direct numerical simulations to understand changes in turbulent Taylor-Couette flow in the presence of riblets [25]. They reported that the presence of the riblets in the Taylor-Couette flow enhanced the transport of momentum through plume ejections that occurred near the tip of the riblets. In their calculations, the riblet surfaces increased the value of the integrated torque acting on the cylindrical rotor compared with the smooth baseline case. However, they also foresaw the possibility of a reduction in torque, if there were no plume ejections from the tips and the grooves acted to impede the Taylor vortices [25].

More recently, Rosenberg et al. used a Taylor-Couette setup with a radius ratio of $R_{\mathrm{i}} / R_{\mathrm{O}}=0.93$, consisting of a rotating outer cylinder and a fixed inner cylinder patterned with liquid-infused streamwise grooves, to study drag reduction using liquid-infused hydrophobic surfaces. Experiments over a range of Reynolds numbers spanning $6000<\mathrm{Re}_{d}<9000$ showed a maximum drag reduction of $14 \%$. These liquid-infused surfaces consisted of $\mathrm{V}$-groove textures on the inner cylinder with spacing of $100 \mu \mathrm{m}$ and height of about $80 \mu \mathrm{m}$ which were filled with various low-viscosity water-immiscible oils [22].

In the present paper we use the Taylor-Couette geometry, specifically in the Couette flow (CF) and the Taylor vortex flow (TVF) regimes [15], to investigate changes in the frictional torque exerted on the inner cylinder as a function of the speed of rotation when the surface texture of the inner cylinder is varied to introduce streamwise V-groove riblets. First, in Sec. II, we briefly review theoretical expressions for the velocity field and torque exerted during Couette flow, as well as describing how the riblets are added to the flow geometry using rapid prototyping techniques. The experimental configuration of the test cell is discussed in Sec. III and subsequently the results of experiments in the $\mathrm{CF}$ regime are presented in Sec. IV and compared with axisymmetric periodic simulations of the swirling flow near the riblets in Sec. IV A. In Sec. IV B we discuss the more complex three-dimensional changes to the local kinematics induced by riblet textures in the TVF regime plus the resulting changes in the torque as a function of the geometry of the $\mathrm{V}$ grooves 
and again we compare our measurements with time-dependent, three-dimensional viscous flow simulations.

\section{TAYLOR-COUETTE FLOW AND RIBLETS: BACKGROUND AND THEORY}

Taylor-Couette flow is the generic name given to the flow generated between two concentric rotating cylinders; in the most general form, both cylinders can rotate independently. Depending on the directions that the cylinders rotate with respect to each other, their relative speeds, and the geometry of the cell, various types of flows and instabilities can be observed and have been reported in the literature using phase maps as a function of the two independent Reynolds numbers that can be defined based on the inner and outer cylinder radii $[15,26]$. If the radii and angular velocities of the inner and the outer cylinders are $R_{\mathrm{i}}, \Omega_{\mathrm{i}}, R_{\mathrm{o}}$, and $\Omega_{\mathrm{o}}$, respectively, then the Reynolds number for the flow, based on the velocity of the inner and outer cylinders, and the gap size $\left(d=R_{\mathrm{O}}-\right.$ $R_{\mathrm{i}}$ ) are defined as $\operatorname{Re}_{d, \mathrm{i}}=\rho R_{\mathrm{i}} \Omega_{\mathrm{i}}\left(R_{\mathrm{o}}-R_{\mathrm{i}}\right) / \mu$ and $\operatorname{Re}_{d, \mathrm{o}}=\rho R_{\mathrm{o}} \Omega_{\mathrm{o}}\left(R_{\mathrm{o}}-R_{\mathrm{i}}\right) / \mu$. In addition to the Reynolds numbers which characterize the dynamics of the flow, two purely geometric dimensionless groups are used to define the geometry of the cell. The radius ratio $\eta=R_{\mathrm{i}} / R_{\mathrm{o}}$ offers a measure of the curvature of the Taylor-Couette cell and the dimensionless length $\Gamma=L /\left(R_{\mathrm{O}}-R_{\mathrm{i}}\right)$ defines the number of Taylor vortices that are established in the annular gap, where $L$ is the axial height of the cell. In the earlier literature this parameter is also often referred to as the aspect ratio (of the TC cell); however, to avoid confusion with the aspect ratio of the riblets introduced later, for clarity we refer to $\Gamma$ as the length-to-gap ratio. In the ideal Taylor-Couette flow, $\Gamma \rightarrow \infty$. To take the effects of streamline curvature and the fluid inertia into account, sometimes instead of a Reynolds number it is convenient to define the Taylor number Ta $=2(1-\eta) /(1+\eta) \operatorname{Re}_{d, \mathrm{i}}^{2}$ [27]. In the present study, we use the Taylor number to extract the critical transition point between the CF and TVF regimes from the table in Ref. [27].

In the present work we focus on the case where only one of the cylinders is rotating at a time. Since the test cell used in this work is attached to a stress-controlled rheometer (discussed later), we can readily impose a torque to the inner cylinder and then measure the resulting angular velocity. Alternatively, one can extend this method to use a strain-controlled rheometer in which an angular velocity $\Omega_{\mathrm{o}}$ is imposed on the outer cylinder while the resulting torque is measured on the inner cylinder. Therefore, out of the wide variety of possible configurations for this flow, the case of a rotating inner cylinder (finite $\operatorname{Re}_{d, \mathrm{i}}$ ) with a fixed outer cylinder $\left(\operatorname{Re}_{d, \mathrm{o}}=0\right)$ and the case of a rotating outer cylinder (finite $\left.\operatorname{Re}_{d, \mathrm{o}}\right)$ with a stationary inner cylinder $\left(\operatorname{Re}_{d, \mathrm{i}}=0\right)$ can most easily be employed.

These two cases become identical at low Reynolds numbers and small gaps $\left(d / R_{\mathrm{i}} \ll 1\right.$ or $\left.\eta \rightarrow 1\right)$ and both configurations are commonly used in rheometric characterization [28]. However, at sufficiently large Reynolds numbers, the case with a rotating inner cylinder (finite $\operatorname{Re}_{d, \mathrm{i}}$ ) and a stationary outer cylinder experiences centrifugal instabilities [15,26,29-31] that result in a sequence of flow transitions from laminar CF to TVF, followed at higher Reynolds number by wavy vortex flow (WVF), then turbulent Taylor vortices, and ultimately the development of featureless turbulence. On the other hand, when only the outer cylinder is rotating, no instability is observed until the transition from laminar to featureless turbulent flow occurs. In the laminar CF regime when only the inner cylinder is rotating, the tangential velocity profile can be written in the form

$$
\frac{v_{\phi}}{R_{\mathrm{i}} \Omega_{\mathrm{i}}}=\frac{\eta}{1-\eta^{2}}\left(\frac{R_{\mathrm{o}}}{r}-\frac{r}{R_{\mathrm{o}}}\right)
$$

When only the inner cylinder is rotating the magnitude of the shear strain rate at the inner cylinder (at $r=R_{\mathrm{i}}$ ) is $\dot{\gamma}_{\mathrm{r} \phi}=\dot{\gamma}_{\phi \mathrm{r}}=2 \Omega_{\mathrm{i}} /\left(1-\eta^{2}\right)$. Thus, for a Newtonian fluid, the only nonzero components of the deviatoric stress tensor acting on the inner cylinder (and thus the magnitude of the traction 
vector at the wall) can be written as

$$
\tau_{\mathrm{w}, 0}=\tau_{\mathrm{r} \phi}=\tau_{\phi \mathrm{r}}=\frac{2 \mu \Omega_{\mathrm{i}}}{1-\eta^{2}} .
$$

On a smooth cylindrical rotor with wall radius located at $r_{\mathrm{w}}=R_{\mathrm{i}}$ the magnitude of the viscous circumferential force and the total torque acting about the axis of rotation are then found as

$$
F_{\phi, 0}=\frac{2 \mu}{1-\eta^{2}} \Omega_{\mathrm{i}}\left(2 \pi R_{\mathrm{i}} L\right)
$$

and

$$
\mathcal{T}_{0}=\frac{2 \mu}{1-\eta^{2}} \Omega_{\mathrm{i}}\left(2 \pi R_{\mathrm{i}}^{2} L\right)=4 \pi v^{2} \rho L \frac{\eta}{\left(1-\eta^{2}\right)(1-\eta)} \operatorname{Re}_{d, \mathrm{i}}
$$

In Eq. (4) the frictional torque is rearranged as a product of a dimensional prefactor, a dimensionless group based on the radius ratio $\left(\eta=R_{\mathrm{i}} / R_{\mathrm{o}}\right)$, and the Reynolds number of the flow. It is thus common to nondimensionalize the total torque by the prefactor $4 \pi \rho \nu^{2} L$ and define the dimensionless torque as $G=\mathcal{T} / 4 \pi \rho v^{2} L$ [32,33]. For the case of a smooth rotor in the laminar regime the dimensionless torque can then be compactly written as

$$
G_{0}=\frac{\eta}{\left[\left(1-\eta^{2}\right)(1-\eta)\right]} \operatorname{Re}_{d, i}
$$

Since in the present work we only examine the case where the inner cylinder is rotating, from this point forward the Reynolds number (denoted for simplicity by $\mathrm{Re}_{d}$ ) will be the Reynolds number using the velocity $\left(v_{\mathrm{i}}=\Omega_{\mathrm{i}} R_{\mathrm{i}}\right)$ of the inner cylinder. For the case of nonsmooth rotors, after computing the stress tensor $\boldsymbol{\tau}$ and then calculating the traction vector $\boldsymbol{\tau}_{\mathrm{w}}=\boldsymbol{\tau}\left(r=R_{\mathrm{i}}, \phi, z\right) \cdot \boldsymbol{n}_{\mathrm{w}}$ on the inner cylinder, we can write quite generally

$$
\begin{aligned}
& F_{\phi}=\int_{S_{\mathrm{w}}} \tau_{\mathrm{w}, \phi} d S_{\mathrm{w}}=\int_{0}^{L} \tau_{\mathrm{w}, \phi} 2 \pi r_{\mathrm{w}} d z, \\
& \mathcal{T}=\int_{S_{\mathrm{w}}}\left[\boldsymbol{r}_{\mathrm{w}} \times \boldsymbol{\tau}_{\mathrm{w}}\right] d S_{\mathrm{w}}=\int_{0}^{L}\left[\boldsymbol{r}_{\mathrm{w}} \times \boldsymbol{\tau}_{\mathrm{w}}\right] 2 \pi r_{\mathrm{w}} d z,
\end{aligned}
$$

where $\boldsymbol{r}_{\mathrm{w}}(z)$ is the local vector determining the position of the wall (with magnitude $r_{\mathrm{w}}$ ) and $\tau_{\mathrm{w}, \phi}$ is the meridional component of $\boldsymbol{\tau}_{\mathrm{w}}$ (see Eq. (13) below). This general form enables us to calculate the torque acting on an arbitrary textured or untextured rotor. For the case of periodic streamwise riblets aligned in the circumferential direction, the local vector $\boldsymbol{r}_{\mathrm{W}}(z)$ is only a function of the axial position $z$. The resulting torque obtained from a numerical simulation or a rheometric measurement with a textured rotor may be larger or smaller than the value given by Eq. (4). Thus the reference torque $\mathcal{T}_{0}$ [defined in Eq. (4)] can be used to calibrate a specific test cell and then used to assess drag reduction (or increase) associated with the addition of textured features by evaluating the general expression given in Eq. (7) and comparing the value with Eq. (4).

When the inner rotor is covered with riblets aligned in the circumferential (flow) direction and covering the entire axial length of the rotor, we need to define additional dimensionless parameters that characterize the dimensions of the riblets. In the present work we focus on $\mathrm{V}$ grooves, which are the simplest riblet geometry. Figure 1(b) shows a schematic of the cross section of a Taylor-Couette cell with streamwise $\mathrm{V}$-shaped riblets on the inner rotor. Two parameters fully define the shape of the riblets: the axial wavelength or spacing of the riblets, here denoted by $\lambda$, and the amplitude of the riblets $A$ [Fig. 1(b)]. In this work the dimensions of the riblets are chosen in a way that $A<R_{\mathrm{i}}$ and $\lambda<R_{\mathrm{i}}$.

As a measure of the sharpness of the $\mathrm{V}$ groove, we use the dimensionless aspect ratio $\mathcal{R}$, defined as $\mathcal{R}=A /(\lambda / 2)=\tan \theta$, to be consistent with our previous works [12,13]. Here $\theta$ is the side angle of the $\mathrm{V}$ groove as shown in Fig. 1(b). Note that for a smooth rotor $\mathcal{R}=0$ and the radius of the wall 


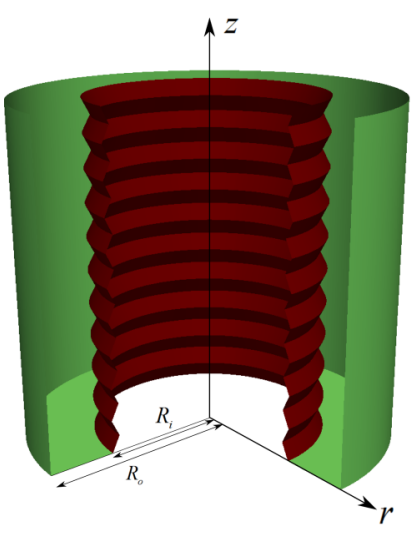

(a)

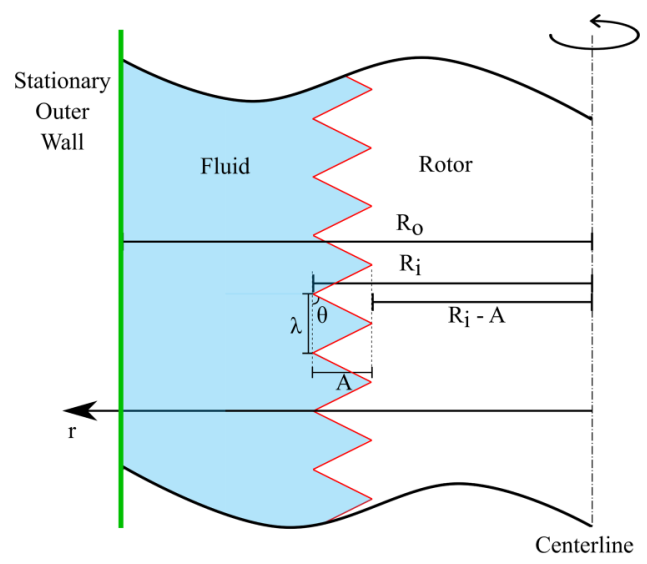

(b)

FIG. 1. (a) Schematic of a Taylor-Couette cell with a riblet-covered inner rotor (red) and smooth stator (green). The inner radius $R_{\mathrm{i}}$ here corresponds to the distance from the center of the coordinate system to the farthest point on the riblets (riblet peaks) and $R_{\mathrm{o}}$ is the radius of the outer cylinder. (b) Enlarged schematic of the riblet geometry on the wall showing the inner and outer radii $R_{\mathrm{i}}$ and $R_{\mathrm{o}}$ as well as the geometry of the riblets. The blue shaded region corresponds to the fluid domain.

on the inner cylinder is $r_{\mathrm{w}}=R_{\mathrm{i}}$ for every axial location $z$. For the case of the riblet-covered inner rotor, the total torque acting on the rotor can be calculated using Eq. (7); however, $r_{\mathrm{w}}$ is no longer a constant and varies in the axial direction. For the case of periodic $\mathrm{V}$-groove riblets we have $r_{\mathrm{w}}=$ $R_{\mathrm{i}}-\mathcal{R} z$ for $0 \leqslant z \leqslant \lambda / 2$ and due to symmetry about the line $z=\lambda / 2$ we have $r_{\mathrm{w}}=R_{\mathrm{i}}-\mathcal{R}(\lambda-z)$ for $\lambda / 2 \leqslant z \leqslant \lambda$.

Due to the closed nature of the streamlines of the flow generated between the two cylinders, this flow is always spatially fully developed without the additional complicating issues relating to Reynolds-number-dependent entrance lengths (as seen in measurements and simulations of pipe or channel flows) [12]. To nondimensionalize the wavelength of the riblets, we use the gap spacing to give $\lambda / d$. This serves as a connection between the riblets' dimensions and the general dimensions of the Taylor-Couette problem. Unlike a boundary layer flow in which the streamwise location along the plate plays an important role and is used for nondimensionalizing the wavelength [12], here the direction of the viscous diffusion in the radial direction (i.e., across the gap) is the more appropriate length scale to be used. Note that using the definition of the radius ratio $\eta=R_{\mathrm{i}} / R_{\mathrm{o}}$, the ratio $\lambda / d$ can also easily be transformed to $\lambda / R_{\mathrm{i}}=\lambda / d[(1-\eta) / \eta]$ if desired.

\section{EXPERIMENTAL SETUP}

A bespoke wide-gap Taylor-Couette cell with a radius ratio of $\eta=0.63$ was designed and built for this work. An image of the test cell is shown in Fig. 2(a). The fixed stator is machined using a computer numerical control (CNC) mill (with a precision of approximately $100 \mu \mathrm{m}$ ) and sits on the fixed Peltier plate of a stress-controlled rheometer (TA Instruments AR-G2). Streamwisealigned riblet-covered rotors with $\mathrm{V}$ grooves were three-dimensional (3D) printed using a Form 1+3D printer and standard resins. The fixed cell and the rotors all have a radius ratio of $\eta=$ 0.63 with $R_{\mathrm{i}}=14 \mathrm{~mm}, R_{\mathrm{O}}=22.23 \mathrm{~mm}$, and $L=42 \mathrm{~mm}$ corresponding to a length-to-gap ratio of $\Gamma=5.1$. The riblets of the rotors are aligned in the circumferential flow direction and they are printed with three different wavelengths of $\lambda=1,2$, and $3 \mathrm{~mm}$ and with three different aspect ratios of $0.58,1$, and 1.73 corresponding to side angles of $\theta=30^{\circ}, 45^{\circ}$, and $60^{\circ}$, respectively. This corresponds to nine different rotors plus one additional smooth rotor $(\mathcal{R}=0)$. (The maximum available resolution in the specifications of the Form $1+$ printer is $300 \mu \mathrm{m}$ and attempts in printing 


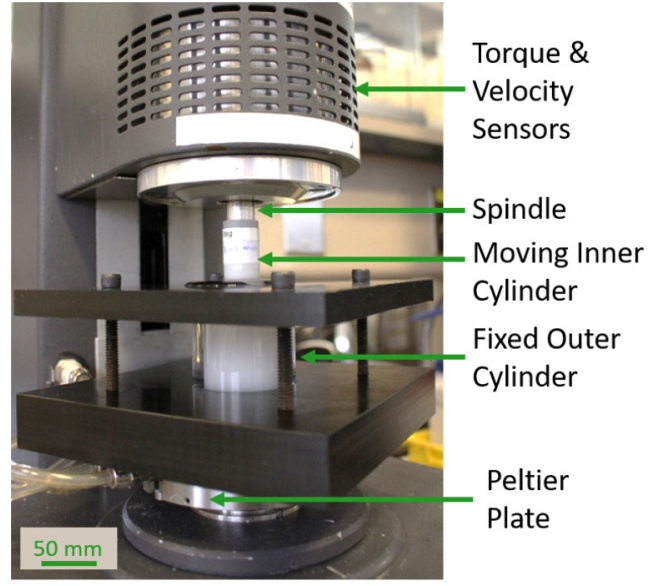

(a)

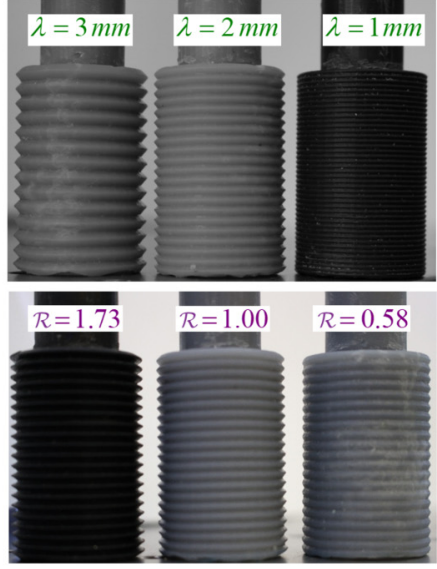

(b)

FIG. 2. (a) Configuration of the custom-designed Taylor-Couette cell used in this study. The Taylor-Couette cell is mounted on the Peltier base of a stress-controlled rheometer (TA Instruments AR-G2). (b) 3D printed riblet-covered rotors with different wavelengths $\lambda=1,2$, and $3 \mathrm{~mm}(\mathcal{R}=1)$ and aspect ratios $\mathcal{R}=0.58,1$, and 1.73 (for fixed wavelength $\lambda=2 \mathrm{~mm}$ ).

riblet-covered rotors with a side angle larger than $70^{\circ}$ were not successful.) The design of the rotors includes a hole at the top of the central shaft which is tapped after the printing process to match the threads at the end of the draw rod located inside the rheometer spindle and allows for the rotors to be mounted and aligned concentrically to the rheometer. For more details regarding the test cell dimensions, precision, and assembly see [14].

To keep the reference flow field outside the riblets as similar as possible to its smooth counterpart, here we place the peak of the riblets at $R_{\mathrm{i}}$ corresponding to the radius of the smooth rotor and the valley of the riblets is located at $R_{\mathrm{i}}-A$. This geometric choice ensures that in the TVF regime the smooth and riblet-covered cases have the same number of vortex pairs, as will be shown in Sec. IV B. Other variations in the geometric choices for the placement of textures can be hypothesized to have an effect on the transition between flow types as well as the flow dynamics, but is beyond the scope of this investigation.

After mounting to the freely rotating spindle of the rheometer, the angular velocity and torque on the inner rotor are measured using contactless sensors located in the rheometer head. The overall assembled Taylor-Couette cell and a rotor as attached to the rheometer spindle are shown in Fig. 2(a) and a selection of the 3D printed rotors are shown in Fig. 2(b).

In all of the experiments the torque was varied over a range of $0<\mathcal{T}<800 \mu \mathrm{N} \mathrm{m}$ and the corresponding angular velocity (over the range of $1 \leqslant \Omega_{\mathrm{i}} \leqslant 120 \mathrm{rad} / \mathrm{s}$ ) was recorded for both smooth and riblet-covered rotors. The procedure used in the experiment consists of a series of "peak hold" steps in which the rheometer is set to apply a fixed torque for a duration of $120 \mathrm{~s}$ while the rotation rate of the inner rotor is measured over 12 increments of $10 \mathrm{~s}$ each. To remove the transient effect of the motor startup at the very beginning of the procedure, the first peak hold step is repeated two times and the first one is ignored. To cover a wider range of Reynolds numbers, the viscosity of the working fluid was also adjusted by using different mixtures of glycerol and deionized (DI) water. The torque and velocity results were then extracted and transformed to Reynolds number and dimensionless torque using the definitions in Sec. II for additional analysis. The location of the transition from CF to TVF is identified using the slope of the dimensionless torque as a function of the Reynolds number and additional discussion can be found in the Supplemental Material [34]. 


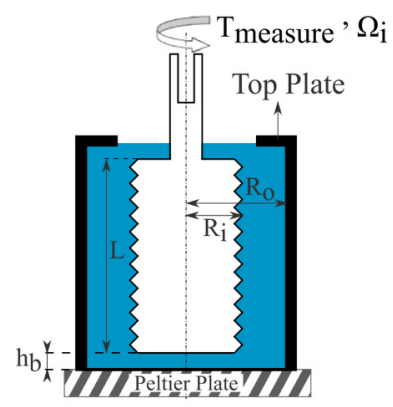

(a)

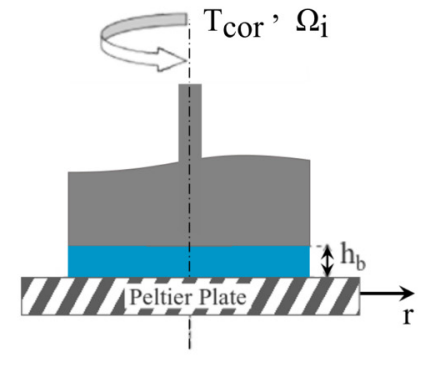

(b)

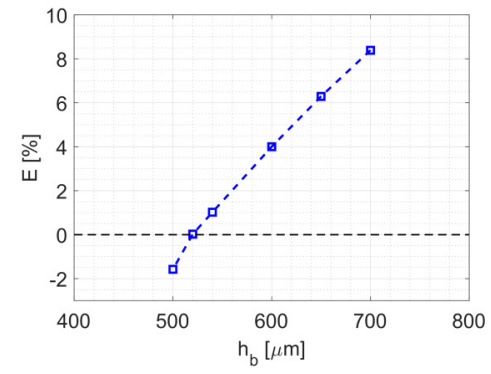

(c)

FIG. 3. (a) Schematic of the geometry of the Taylor-Couette setup with closed bottom gap offset by height $h_{\mathrm{b}}$. (b) Schematic of the torsional flow between the stationary bottom of the Taylor-Couette cell and the bottom of the rotor. (c) Calibration plot, showing the error as defined in Eq. (9) as a function of the bottom gap height $h_{\mathrm{b}}$ at a fixed Reynolds number $\operatorname{Re}_{d}=4$.

\section{Calibrating the effect of the closed ends}

The most common experimental imperfection in any Taylor-Couette setup arises from the added torque arising due to the no-slip boundary condition acting at the bottom enclosure of the cell and the bottom of the rotor. Depending on the specifics of the test configuration used, similar conditions can also arise at the top of the cell [28].

In the present setup, the rotors are connected to the head of the rheometer through the spindle and are immersed in the fluid inside the Taylor-Couette cell as shown in Fig. 3(a). The rotor does not come into contact with the bottom of the cell but is axially offset by a distance $h_{\mathrm{b}}$. As shown in Fig. 3(a), while the Taylor-Couette cell has a top plate, the opening of this plate has a diameter larger than the diameter of the rotors used. Therefore, the top of the rotor is only in contact with fluid. The shear-free nature of the resulting boundary condition means negligible additional frictional effect is contributed from the top of the rotor.

At the closed bottom of the Taylor-Couette cell the additional torque from the torsional shearing flow induced between the rotor and stator leads to a systematic deviation of the torque above the values calculated from creeping flow theory given in Eqs. (4) and (7). We decompose the experimentally measured torque (denoted by $\mathcal{T}_{\text {expt }}$ ) into two combinations $\mathcal{T}_{\text {expt }}=\mathcal{T}_{0}+\mathcal{T}_{\text {cor }}$, i.e., the theoretical part, given by Eq. (4), and a correction term $\mathcal{T}_{\text {cor }}$, which is expected to vary with the rotor size.

Different methods can be employed to find $\mathcal{T}_{\text {cor }}$. For example, Rosenberg et al. first make their measurements using a Taylor-Couette cell of $\eta=0.93$ and $\Gamma=40$ (with a textured, fixed inner rotor and rotating outer cylinder) completely filled with fluid (over a range of $1500<\mathrm{Re}_{d}<9000$ ) corresponding to the total measured torque $\mathcal{T}_{\text {expt }}$. Then they repeat the experiments, with the same Taylor-Couette cell, but this time only partially filled with fluid up to the bottom of the rotor to determine the end correction $\mathcal{T}_{\text {cor }}$. They then subtract this result from the original torque measurements $\mathcal{T}_{\text {expt }}$ to find the true torque in the absence of end effects [22].

Here, to find the correction term $\mathcal{T}_{\text {cor }}$, we model the additional effect of the viscous flow between the bottom of the Taylor-Couette cell and the bottom of the rotor as a torsional shear flow between two parallel plates, as shown in the schematic in Fig. 3(b). Therefore, this correction term can be analytically calculated and written as [28]

$$
\mathcal{T}_{\text {cor }}\left(h_{\mathrm{b}}\right)=\frac{\pi R_{\mathrm{i}}^{3} \mu}{2}\left(\frac{\Omega_{\mathrm{i}} R_{\mathrm{i}}}{h_{\mathrm{b}}}\right) .
$$

For a Taylor-Couette cell with a fixed geometry, the term $\mathcal{T}_{\text {cor }}$ is a function of the bottom gap separation, denoted by $h_{\mathrm{b}}$. Now with Eq. (8), one can calibrate the experimental setup for the gap 


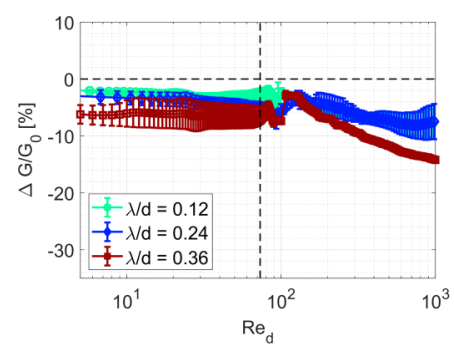

(a)

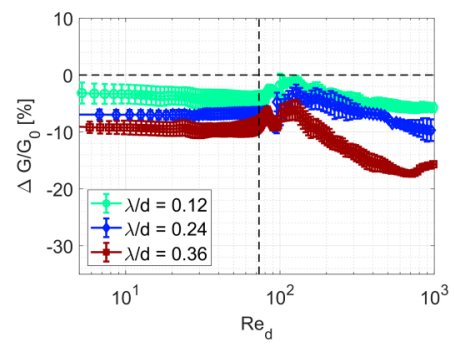

(b)

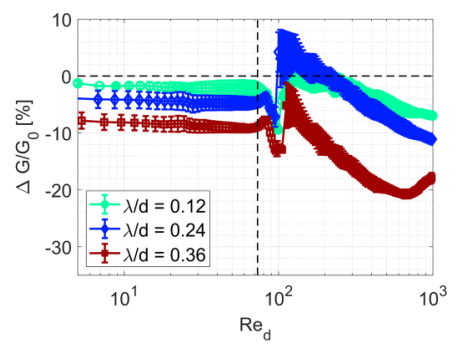

(c)

FIG. 4. Results of reduction in torque measured for riblet-covered rotors compared with smooth rotors as a function of the Reynolds number and $\lambda / d$ for (a) $\mathcal{R}=0.58$, (b) $\mathcal{R}=1.00$, and (c) $\mathcal{R}=1.73$.

separation $h_{\mathrm{b}}$ that accounts for the equality $\mathcal{T}_{\text {expt }}=\mathcal{T}_{0}+\mathcal{T}_{\text {cor }}$. In the calibration, we use a mixture of $75 \%$ glycerol with $25 \%$ DI water at a small fixed Reynolds number of $\operatorname{Re}_{d}=4$ and the torque on the inner rotor is measured while changing the bottom gap $h_{\mathrm{b}}$ and then the results are compared with the calculated torque using $\mathcal{T}_{0}+\mathcal{T}_{\text {cor }}$. The difference between the measured and calculated torques define the percentage of extra stress arising from the end,

$$
E\left(h_{\mathrm{b}}\right)=\frac{\mathcal{T}_{\text {expt }}-\left(\mathcal{T}_{0}+\mathcal{T}_{\text {cor }}\right)}{\mathcal{T}_{0}+\mathcal{T}_{\text {cor }}} \times 100,
$$

as a function of the gap height $h_{\mathrm{b}}$ and the results are shown in Fig. 3(c). As shown in the figure, a bottom gap separation of $h_{\mathrm{b}}=520 \mu \mathrm{m}$ gives almost no error $(0.02 \%)$ and hence this gap height has been held constant and is used for the rest of the measurements. For the range of $3<\operatorname{Re}_{d}<60$ the $L_{\infty}$ norm of the error for the measurements shown later [in Fig. SI1(a) in the Supplemental Material [34]] is found to be $0.9 \%$ and for $60<\mathrm{Re}_{d}<80$, which is in the transition region, the norm of the error increases with $\operatorname{Re}_{d}$ but does not exceed $3.5 \%$.

\section{EXPERIMENTAL RESULTS}

Since we are interested in understanding the effects of the riblet geometry on the changes in the total frictional torque acting on the inner cylinder, our results are reported in the form of net changes in torque as a function of the various dimensionless variables introduced above. First, we summarize the results of all experiments over a range of Reynolds numbers of $4<\operatorname{Re}_{d}<1000$ in Fig. 4. Here the percentage changes in torque are defined as

$$
\frac{\Delta G}{G_{0}}=\frac{G-G_{0}}{G_{0}} \times 100,
$$

where $G$ is the nondimensional torque measured for the riblet-covered rotors and corrected using Eq. (8) and $G_{0}$ is the nondimensional torque exerted on the corresponding smooth rotor, also after end correction [see Fig. SI1(a) in the Supplemental Material [34]]. The results are presented as a function of the measured Reynolds number $\operatorname{Re}_{d}$, the aspect ratio $\mathcal{R}$ of the riblets, and the dimensionless wavelength $\lambda / d$.

The vertical dashed line shows the location of the theoretical transition point $\operatorname{Re}_{\text {crit }}=74$ to TVF. The horizontal dashed line shows a baseline corresponding to no net change in torque. Based on the definition of the torque change in Eq. (10), a negative torque change $(\Delta G<0)$ corresponds to a frictional torque reduction and a positive torque change corresponds to an increase in the frictional torque acting on the grooved rotor. Each experiment was repeated at least three times to confirm repeatability as well as to obtain the standard deviation (error bars) shown in the figures. The data reported between Reynolds numbers of $4<\operatorname{Re}_{d}<105$ were measured using a working fluid composed of $75 \%$ by volume glycerol and $25 \%$ DI water. This choice was guided 
by the reports of previous experiments with millimeter-sized riblets [24,35] where instead of water, oil (with a larger kinematic viscosity) was used. Additional discussion can be found in the Supplemental Material [34]. Because of the sensitivity of the viscosity to composition, the dynamic viscosity of the mixture was remeasured (before and after each test, to adjust for any possible viscous heating or change in temperature) and the mean of the experimental value was subsequently used for the data analysis. In addition, it can be shown that the Nahme-Griffith number comparing viscous heating arising from shear flow in the gap and radial heat conduction is always smaller than $\mathrm{Na} \leqslant 0.023$ (i.e., much smaller than unity) and thus has no effect on the results. More information can be found in the Supplemental Material [34]. The range of data between $100<\operatorname{Re}_{d}<1000$ was measured using a working fluid with $50 \%$ by volume of glycerol and $50 \%$ DI water to avoid air entrapment next to the rotating shaft (additional discussion is in the Supplemental Material [34]) and again for each test the viscosity of the fluid was measured and used for the analysis.

\section{A. Cylindrical Couette flow regime}

In a laminar steady unidirectional flow $(\mathrm{CF})$ in which only the inner cylinder is rotating, prior to transition to TVF, the velocity profile is expected to only be a function of the geometry of the Taylor-Couette cell and not a function of the Reynolds number [Eq. (1)]. A similar invariance is expected even in the presence of riblets and therefore it is expected that the changes in the torque induced by the riblets will not be a function of the Reynolds number prior to transition to TVF. Figure 4 indeed shows that before exceeding the critical Reynolds number, the changes in the torque are nearly constant with respect to $\mathrm{Re}_{d}$. While all cases show a torque reduction, the change in torque is only dependent on changes in the riblet geometry and not on the dynamics of the flow (for $\operatorname{Re}_{d}<\operatorname{Re}_{\text {crit }}$ ).

To confirm this experimental observation, axisymmetric periodic models of a single riblet in a Taylor-Couette geometry with the same wavelength $\lambda / d$ and a range of aspect ratios $0<\mathcal{R}<2.5$ were created and numerical simulations were performed using the OpenFOAM finite-volume solver. We used the SIMPLE algorithm to compute the velocity field for steady-state axisymmetric TaylorCouette flow with periodic boundary conditions at $z=0$ and $z=\lambda$. We calculated the changes in torque for a range of $0<\mathcal{R}<2.5$ and $\lambda / d$ values similar to those used in the experiments. To compare the effect of changes in the Reynolds number, two cases with $\operatorname{Re}_{d}=23$ and 58 were modeled. In the corresponding sets of experiments up to $10 \%$ torque reduction was observed in the $\mathrm{CF}$ regime. As seen in Fig. 5, the measured and calculated changes in torque are the same for both values of the Reynolds number computed as long as the dimensionless variables characterizing the riblet geometry are kept constant (as also seen in Fig. 4). However, varying the geometry of the riblets by either changing the $\mathcal{R}$ or changing $\lambda / d$ has a direct effect on the frictional torque acting on the rotor.

We first consider the case where the wavelength of the riblets is kept constant and the amplitude of the riblets is varied (changing $\mathcal{R}$ ). We replot the results extracted from Fig. 4 at $\operatorname{Re}_{d}=23$ and 58 as shown in Figs. 5(a)-5(c). It is clear that for a constant value of $\lambda / d$ the change in torque is a nonmonotonic function of the aspect ratio $\mathcal{R}$ of the riblets. The maximum torque reduction happens at an intermediate aspect ratio which varies with the wavelength. In addition, as the wavelength $\lambda / d$ is increased, a wider range of aspect ratios offers torque reduction.

Since the design of the riblets result in the valley walls of the entire groove geometry having a moment arm slightly smaller than $R_{\mathrm{i}}$ as shown in Fig. 1(b), using the computational results, we also present the changes in the circumferential force exerted on the rotor compared to the case of the smooth rotor as shown in Fig. 5(d). While the magnitude of the moment $\operatorname{arm} r_{\mathrm{w}}(z)$ along the riblet-covered rotors is always lower than or equal to the case of the smooth rotor, Fig. 5(d) confirms that the total frictional torque reduction achieved is only partially due to the reduction of the local moment arm and additional contributions arise from changes to the local flow near the 


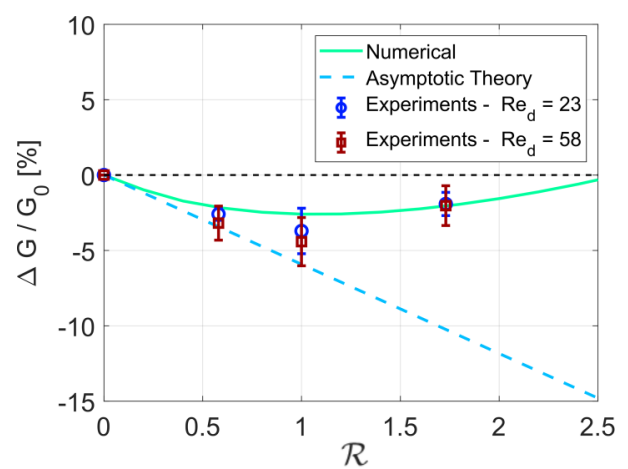

(a)

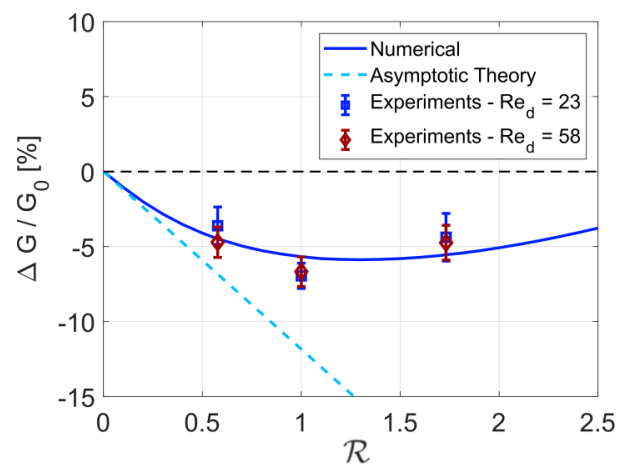

(c)

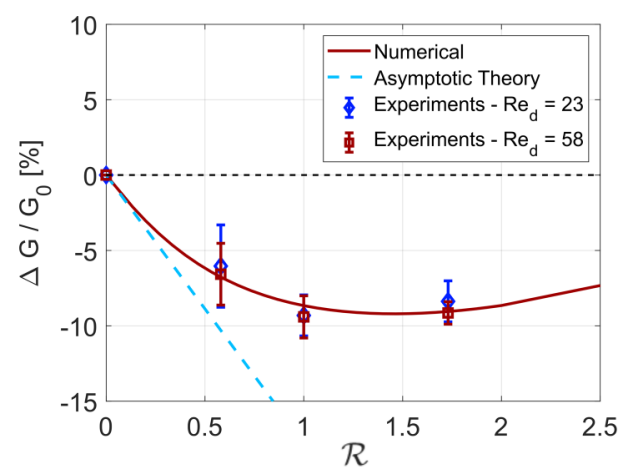

(b)

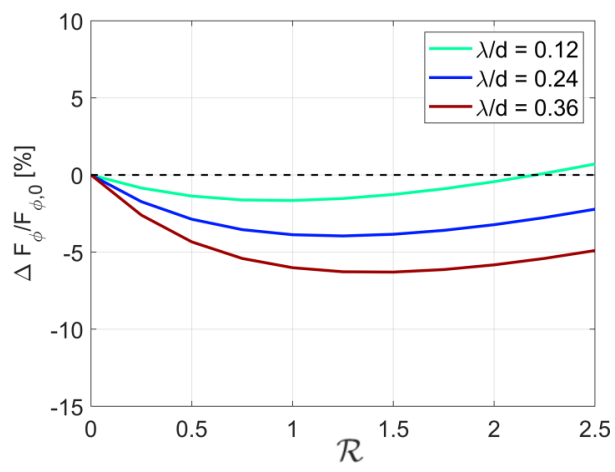

(d)

FIG. 5. Numerical calculation, theoretical prediction, and experimental measurements of the change in torque $\Delta G$ for $\operatorname{Re}_{d}=23$ and $\operatorname{Re}_{d}=58$ as a function of the riblet aspect ratio $\mathcal{R}$ for (a) $\lambda / d=0.12$, (b) $\lambda / d=$ 0.24 , and (c) $\lambda / d=0.36$. (d) Changes in circumferential force exerted on the rotor of a Taylor-Couette cell with $\eta=0.63$ as a function of the aspect ratio of the riblets with $\lambda / d=0.12,0.24$, and 0.36 .

riblets. Further discussion on the choices for the reference smooth rotor used for comparison can be found in the Supplemental Material [34].

In the limit of very shallow riblets, i.e., $2 A / R_{\mathrm{i}} \ll 1$, we can employ an asymptotic expansion of the velocity profile along with a domain perturbation analysis to theoretically calculate the changes in torque due to the riblet surfaces which can be written as

$$
\frac{\Delta G}{G_{0}} \cong-\frac{1}{2} \mathcal{R}\left(\frac{\lambda}{d}\right)\left(\frac{1}{\eta(1+\eta)}\right)+O\left(\mathcal{R}^{2}\left(\frac{\lambda}{R_{\mathrm{i}}}\right)^{2}\right)+\cdots
$$

Details of the analysis can be found in Appendix A and [14]. This theoretical prediction is shown with the dashed blue line in Figs. 5(a)-5(c) for $\eta=0.63$. As can be seen in the figures, this asymptotic result captures the initial slope in the variation of $\Delta G / G_{0}$ as $\mathcal{R} \rightarrow 0$ for all three cases of $\lambda / d=0.12,0.24$, and 0.36 . Also, as expected, Eq. (11) confirms that the riblet-induced changes in torque during steady CF are independent of the Reynolds number. However, at larger aspect ratios $(\mathcal{R}>0.1)$ the asymptotic theory fails to predict the progressive torque increase (i.e., nonmonotonic behavior of the changes in torque) seen from both the numerical and experimental results at high $\mathcal{R}$.

The nonmonotonic behavior of the change in frictional torque as a function of the aspect ratio that is documented in Figs. 5(a)-5(c) and 12(a) can be examined in more detail by considering the component of the traction vector $\left(\boldsymbol{\tau}_{\mathrm{w}}=\boldsymbol{\tau} \cdot \boldsymbol{n}_{\mathrm{w}}\right)$ along the wall of the inner rotating cylinder. When 

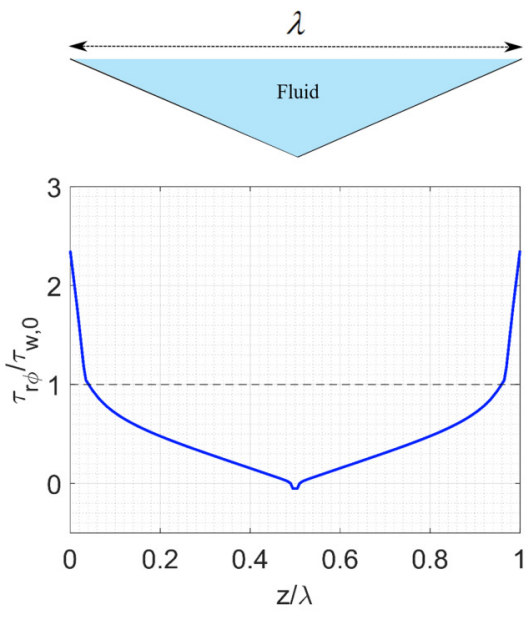

(a)
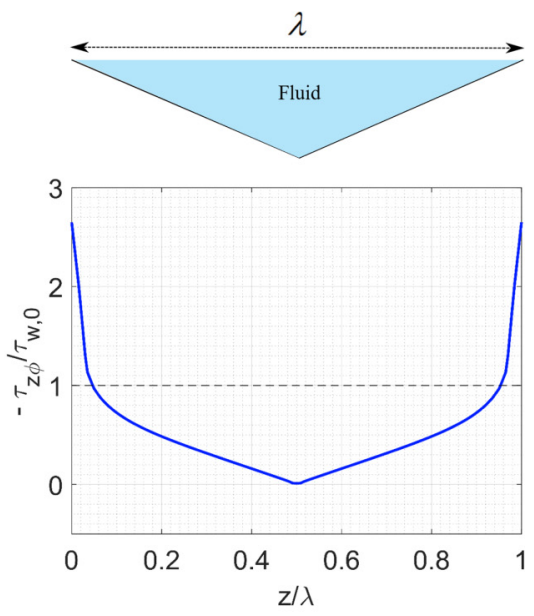

(b)

FIG. 6. Distribution of shear stress components (a) $\tau_{\mathrm{r} \phi}$ and (b) $\tau_{\mathrm{z} \phi}$ normalized by the wall shear stress in the case of a smooth rotor $\left(\tau_{\mathrm{w}, 0}=\tau_{\mathrm{r} \phi, 0}=\tau_{\phi \mathrm{r}, 0}\right)$ as a function of the axial location on the riblet surface with $\lambda / d=0.12$ and $\mathcal{R}=1$, computed at $\operatorname{Re}_{d}=58$.

the riblets are present, the normal vector to the wall $\boldsymbol{n}_{\mathrm{w}}$ can be written as

$$
\boldsymbol{n}_{\mathrm{w}}=\left[\begin{array}{lll}
\cos \theta & 0 & -\sin \theta
\end{array}\right]^{\mathrm{T}}=\left[\begin{array}{ccc}
\frac{1}{\sqrt{1+\mathcal{R}^{2}}} & 0 & -\frac{\mathcal{R}}{\sqrt{1+\mathcal{R}^{2}}}
\end{array}\right]^{\mathrm{T}} .
$$

For a smooth rotor with $\mathcal{R}=0$ the wall normal reduces back to $\boldsymbol{n}_{\mathrm{w}}=\boldsymbol{e}_{\mathrm{r}}$ as expected. Using this expression, the component of the traction vector acting in the circumferential direction which contributes to the frictional torque can be found to be $\tau_{\mathrm{w}, \phi}=\left(\boldsymbol{\tau} \cdot \boldsymbol{n}_{\mathrm{w}}\right) \cdot \boldsymbol{e}_{\phi}=\tau_{\mathrm{r} \phi} \cos \theta-\tau_{\mathrm{z} \phi} \sin \theta$ and thus

$$
\tau_{\mathrm{w}, \phi}=\tau_{\mathrm{r} \phi} \frac{1}{\sqrt{1+\mathcal{R}^{2}}}-\tau_{\mathrm{z} \phi} \frac{\mathcal{R}}{\sqrt{1+\mathcal{R}^{2}}} .
$$

As shown in Sec. II, for a smooth-walled cylinder with $\boldsymbol{n}_{\mathrm{w}}=\boldsymbol{e}_{\mathrm{r}}$ the flow at all $\operatorname{Re}_{d}<\operatorname{Re}_{\text {crit }}$ is a homogeneous shear flow with no axial dependence (i.e., $\tau_{\mathrm{z} \phi}=0$ ) and thus the magnitude of the traction vector at the wall $\tau_{\mathrm{w}, 0}$ is found as in Eq. (2). In the presence of riblets, in addition to $\tau_{\mathrm{r} \phi}$ the stress tensor will also have a nonzero value of $\tau_{\mathrm{z} \phi}$ and both of these shear stress components vary across the riblet surface as shown in Figs. 6(a) and 6(b). The highest shear stress acts at the peaks of the riblets and the lowest shear stress at the trough of the riblets. These stress profiles remain invariant for all $\mathrm{Re}_{d}<\mathrm{Re}_{\text {crit }}$.

The evolution in the circumferential components of the traction vector $\tau_{\mathrm{w}, \phi}$ acting on each riblet as the aspect ratio is varied is shown in Fig. 7(a). Substituting these wall shear stress profiles in Eq. (13) and performing the integration in Eq. (7) over the wavelength $0<z / \lambda<1$ results in the total torque acting on the riblet surface. It is clear from Fig. 7(a) that the introduction of the riblets results in a spatially varying distribution of the circumferential component of the traction vector at the wall. Close to the trough of the riblets the wall shear stress is lower than the value $\tau_{\mathrm{w}, 0}$ expected for the case of the smooth rotor and near the peaks of the riblets $\tau_{\mathrm{w}}$ is locally higher than for the smooth rotor. Similar behavior has been reported previously in flows over riblets in channel flows and boundary layer flows $[4,12,13,36]$.

Closer inspection of the computed stress profiles in Fig. 7(a) shows that as the aspect ratio $\mathcal{R}$ of the riblets is increased, the axial variation of $\tau_{\mathrm{w}}$ evolves so that a progressively wider region of each periodic riblet experiences a wall shear stress which is lower than $\tau_{\mathrm{w}, 0}$. Concomitantly, a 


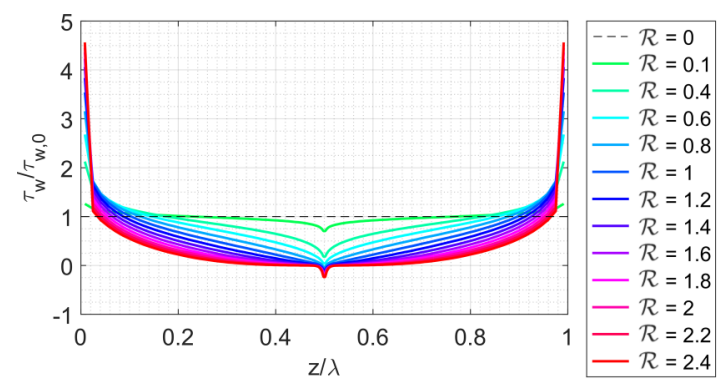

(a)

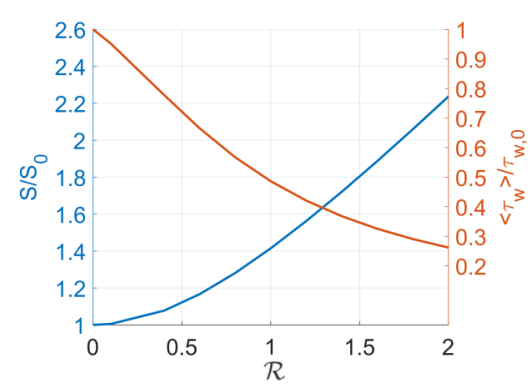

(b)

FIG. 7. (a) Distribution of circumferential component of the wall traction vector as a function of the riblet $\mathcal{R}$ for a single riblet of $\lambda / d=0.12$ at $\operatorname{Re}_{d}=58$ normalized by the circumferential traction of the corresponding case with a smooth rotor. (b) Evolution of the average shear stress for $\operatorname{Re}_{d}=58$ and $\lambda / d=0.12$ as a function of $\mathcal{R}$ and the change in the wetted area $S_{\mathrm{w}}$ of the riblet surface as a function of the aspect ratio. Increasing the aspect ratio of the riblets results in a decrease in the average shear stress acting over one riblet while the wetted area $S$ increases monotonically with $\mathcal{R}$. Here $S_{0}$ is the wetted surface area of a smooth rotor (over the length of a single wavelength) in cylindrical Couette flow, $S_{0}=2 \pi R_{\mathrm{i}} \lambda$.

more-localized region of the riblet near the apex of each riblet experiences a wall shear stress that is larger than the reference value experienced on a smooth rotor. If we define an average shear stress

$$
\left\langle\tau_{\mathrm{w}}\right\rangle=\frac{1}{\lambda} \int_{0}^{\lambda} \tau_{\mathrm{w}}(z) d z
$$

the riblet surface experiences an average shear stress that is lower than that of the smooth rotor $\tau_{\mathrm{w}, 0}$ as defined in Eq. (2). Increasing the aspect ratio $\mathcal{R}$ of the riblets results in a progressive decrease in the average shear stress as shown by the red curve in Fig. 7(b). A similar behavior is also reported in the investigation of the boundary layer flow over riblets [12]. However, adding a texture such as riblets and increasing the aspect ratio of these features also results in a steady increase in the total wetted area of the inner cylinder. This increased trend in the wetted area is also shown in Fig. 7(b). It is this interplay between the decrease in the average shear stress acting on the wall and the increase in the wetted area of the textured surface as the aspect ratio is increasing that results in the nonmonotonic behavior of the total torque as a function of the aspect ratio [shown in Fig. 5(b)]. Thus, using Eq. (7) for V-groove riblets, we have

$$
\begin{aligned}
F_{\phi} & =\int_{0}^{\lambda} \tau_{\mathrm{w}, \phi} d S=\int_{0}^{\lambda} \tau_{\mathrm{w}, \phi} 2 \pi r_{\mathrm{w}} d l=\int_{0}^{\lambda} r_{\mathrm{w}} \tau_{\mathrm{w}, \phi} 2 \pi \sqrt{1+\mathcal{R}^{2}} d z \\
\mathcal{T} & =\int_{0}^{\lambda}\left(\boldsymbol{r}_{\mathrm{w}} \times \boldsymbol{\tau}_{\mathrm{w}}\right) \cdot \boldsymbol{e}_{z} d S=\int_{0}^{\lambda} r_{\mathrm{w}} \tau_{\mathrm{w}, \phi} 2 \pi r_{\mathrm{w}} d l=\int_{0}^{\lambda} r_{\mathrm{w}}^{2} \tau_{\mathrm{w}, \phi} 2 \pi \sqrt{1+\mathcal{R}^{2}} d z .
\end{aligned}
$$

For small aspect ratios, the decrease in the local shear stress on the wall dominates and evaluation of these integrals results in a reduction in the total torque. However, for larger aspect ratios, the increase in the area arising from the last terms in the integrands [Eqs. (15) and (16)] overwhelms the decrease in the average shear stress distribution and the total integrated force and torque increase, until ultimately the addition of riblets no longer offers any reduction in frictional force or torque. The location of the optimal force or torque reduction depends on $\lambda / d$; however, for the range of $\lambda / d$ shown in Fig. 5, it is clear that the optimum aspect ratio is within $1<\mathcal{R}<1.5$. Clearly, due to the specific choice of design in this study, Eq. (16) has an additional $r_{\mathrm{w}}(z)$ factor which results in a slightly larger value of the torque reduction than is obtained in the corresponding force. However, as we have discussed earlier, the total circumferential force exerted on the riblet-covered rotors still experiences a net decrease compared to the smooth rotors and these changes arise from the 
geometry mediated changes in the local viscous flow near the grooves as opposed to only changes in the geometry.

\section{B. Taylor vortex regime}

After transition to the TVF or WVF regime, the structure of the azimuthal velocity profiles becomes dependent on the Reynolds number and thus the resulting measurements of the torque reduction show an additional slight dependence on the Reynolds number. To explore these states we again use experiments in our Taylor-Couette cell mounted on the rheometer, together with time-dependent axisymmetric simulations of the swirling flow $\boldsymbol{v}=\left[v_{r}(r, z ; t), v_{\phi}(r, z ; t), v_{z}(r, z ; t)\right]$ in the TVF regime. As shown in Fig. 4, immediately after transition, within $74<\operatorname{Re}_{d}<110$, the experimental torque measurements show a local speed-dependent increase or decrease as a result of the hysteresis in the vicinity of flow transition, depending on whether the rotor torque is increased from below the transition point to above the transition or vice versa. From Fig. SI1(b) in the Supplemental Material [34] it can also be seen that this transition spans a finite range of rotation rates before the TVF regimes is fully established. Similar hysteresis in the behavior of the TVF was reported previously by Andereck et al. [26]. In addition, as mentioned earlier, since the exponent of the torque does not change up to $\operatorname{Re}_{d} \approx 1000$, we conclude that our experiments have not transitioned from the TVF into the WVF.

In the Taylor vortex regime, in addition to the relative size of the gap (which uniquely controls the velocity and stress profiles in the homogeneous Couette shearing profile), the wavelength of the vortex becomes another important independent length scale in the problem. Various researchers have indicated that this wavelength is not a single invariant fixed value and depends on the flow history used to establish the TVF state [37-39]. Gorman and Swinney have reported observing a variation of between 16 and 20 vortices in a Taylor-Couette apparatus of $\Gamma=20$ [40].

In the present experimental setup, with $\Gamma=5.1$, at steady state three toroidal vortex pairs can clearly be identified. However, in some cases, right after the transition to TVF four vortex pairs are first observed visually and as the flow evolves they ultimately collapse into three stable pairs in the steady-state case. Similar behavior is observed in the results of the numerical simulation with $\operatorname{Re}_{d}=230$ and $\eta=0.63$. Here we select $\Gamma=3.4$ for the simulations $(2 / 3$ of the length of the experimental setup) to reduce the cost of the simulations and eliminate end effects. The simulations are performed in OpenFOAM with the PISO algorithm; the diffusion terms are discretized with a second-order central difference, the convective terms are discretized with a second-order central difference with correction for the nonorthogonality of the mesh faces due to the riblets, and the time integration is performed with a second-order backward Euler scheme.

Initially, three counterrotating vortex pairs are formed and then the vortices interact to adjust their wavelengths and collapse ultimately to two vortex pairs by the time the flow reaches the steady state. Snapshots of the simulated azimuthal velocity profiles alongside the evolution of the torque in the Taylor-Couette geometry are presented in Fig. 8(a). Here time is nondimensionalized by the vorticity diffusion time $t_{d}=d^{2} / v$. It can be seen that near the beginning (point $A$ ) the flow possesses axially uniform characteristics. As time progresses to $t / t_{d} \approx 0.1$ (as shown via points $B$, $C$, and $D$ ), the flow exhibits the first signs of instability and three vortex pairs are initially formed in the flow. It is clear that after the Taylor-Couette flow has transitioned to the TVF regime, the velocity field that was initially only a function of the radial position is now a function of both the radial and axial directions and new vortical structures appear in the flow due to the instabilities that were discussed earlier. However, as we reach $t / t_{d} \approx 0.55$, as shown at points $E, F, G$, and $H$, the three vortices combine to form two vortices which persist as the flow finally approaches steady state corresponding to the constant torque observed for $t / t_{d}>1$. As seen in Fig. 8(a), the temporal evolution of the torque up to the steady-state value goes through different paths with jumps at different times as the vortices form and combine together. Note that the ultimate value of the steady torque recorded in the TVF regime is close to 1.9 times the torque in the CF regime. Similar vortex pair evolution with vortex pairs merging prior to the final steady state, independent of the initial 

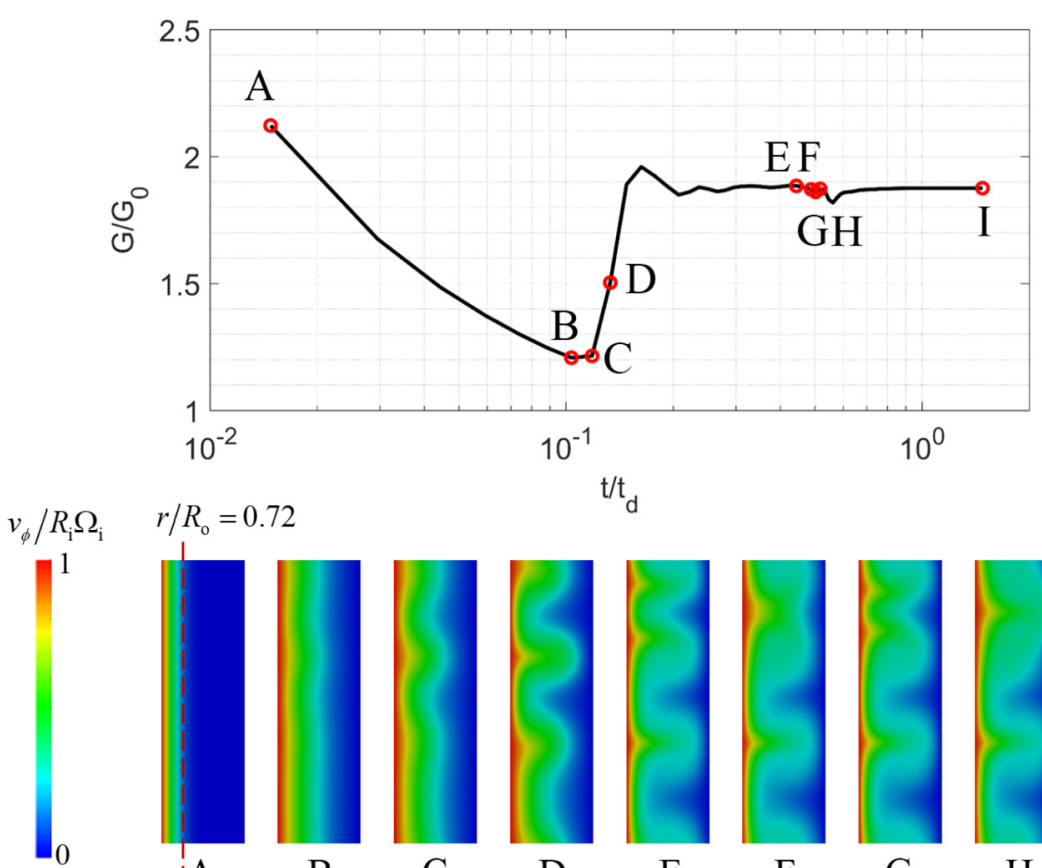

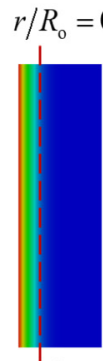

A

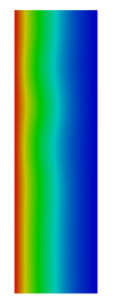

B

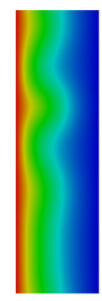

C

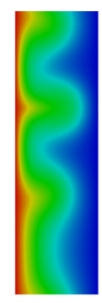

D

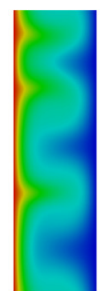

E

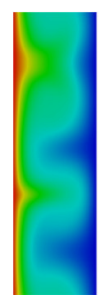

F

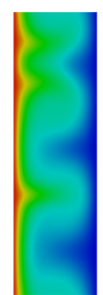

G

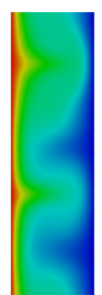

H

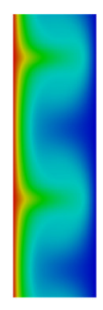

I

(a)

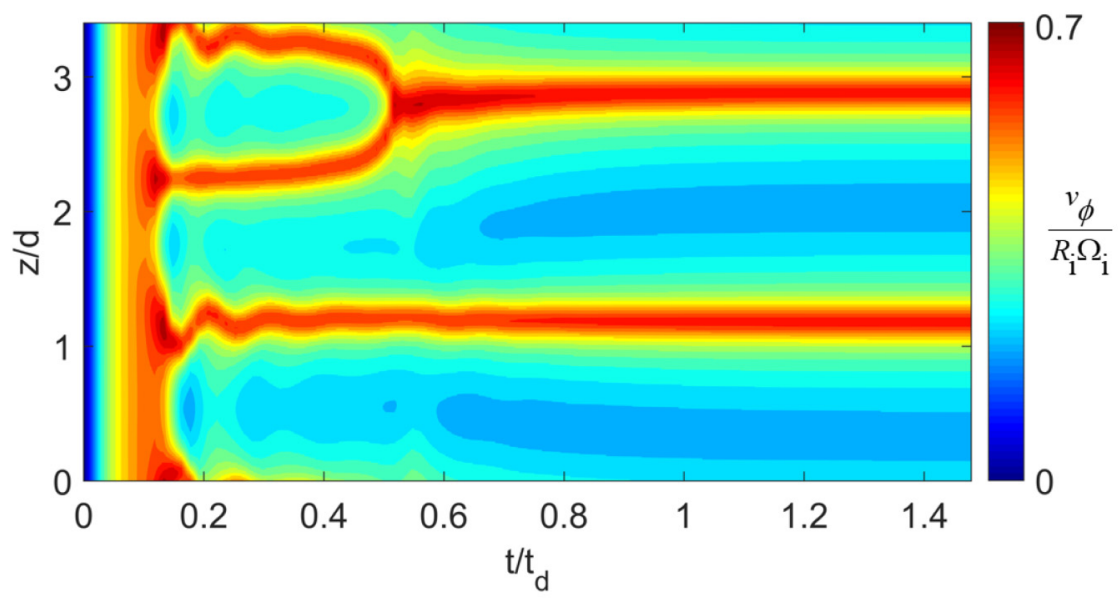

(b)

FIG. 8. (a) Simulation showing the evolution of frictional torque over time during development of TVF at $\operatorname{Re}_{d}=230, \mathrm{Ta}=24016, \eta=0.63$, and $\Gamma=3.4$ for a smooth rotor. The torque is normalized by $G_{0}$ defined in Eq. (5) (the unidirectional base case) and time is normalized by the diffusion time for vorticity $t_{d}=d^{2} / \nu$. Snapshots of the azimuthal velocity at different time instances (denoted by $A-I$ ) are shown in the middle row of images underneath the plot. (b) Space-time evolution of the azimuthal velocity at the radial position $r / R_{\mathrm{o}}=$ 0.72. The axial direction $z$ is nondimensionalized with the gap size $d$ and time is again nondimensionalized with the vorticity diffusion time. 


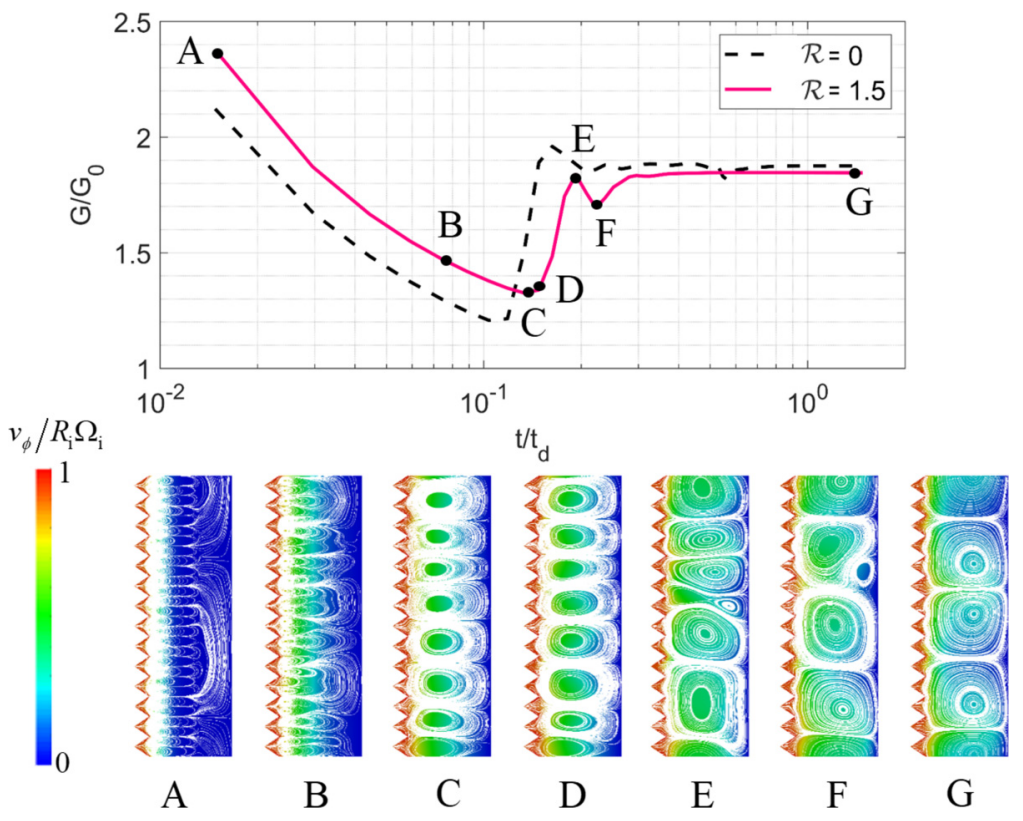

FIG. 9. Time evolution of torque for riblet-covered rotors with $\mathcal{R}=1.5$ and $\lambda / d=0.24$ at $\operatorname{Re}_{d}=230$ compared with the reference case of a smooth rotor. Corresponding contour plots of the azimuthal velocity profile as well as in-plane streamlines with axial and radial velocities are shown for distinct times where various changes occur in the flow. This particular riblet surface can reduce the total drag by about $1 \%$.

condition of the experiments, have also been previously reported for wide-gap $(\eta<0.8)$ TC cells with low height-to-gap ratios $(\Gamma<11)[41-45]$.

Additionally, we present a space-time plot of the evolution of the azimuthal velocity at the radial location $r / R_{\mathrm{o}}=0.72$ in Fig. 8(b). The axial direction $z$ is nondimensionalized with the gap size $d$. It is clear that for early times $0.1<t / t_{d}<0.55$, three vortices can be identified, but subsequently these vortices are combined into two stable cells and then the flow reaches its steady-state form with a constant torque as shown at point $I$ in Fig. 8(a).

In addition to this hydrodynamic instability, the presence of riblets in the TVF also impacts the evolution of the flow and hence the total torque evolves due to a combination of the viscousdominated shearing stress (similar to the case of the CF discussed earlier) coupled with the interaction of the small-scale riblets (especially their peaks) with the larger length scale Taylor vortices that develop in the flow. The general trends observed for the time evolution of the frictional torque on the inner cylinder for Taylor-Couette flow at $\mathrm{Re}_{d}=230$ and each of the riblet-covered rotors with aspect ratios of $0.2 \leqslant \mathcal{R} \leqslant 2$ are very similar; however, the specific values of the torque and critical time for transition to TVF, as well as the final value of the torque, depend on the aspect ratio of the riblets. Drag reduction (compared to the smooth rotor) for all values of $\mathcal{R}<1.6$ can be observed at steady state, whereas drag increase is observed for higher aspect ratios. (Representative data are plotted in the Supplemental Material [34].) Note that no theoretical results for the torque in the Taylor vortex region have been reported to date; however, various functional forms such as power-law scaling have been suggested in different Reynolds-number regimes throughout the years. As mentioned earlier, in the TVF, the measured torque on a smooth rotor varies as $\operatorname{Re}_{d}^{1.54}$, which is very close to the exponent of 1.5 reported earlier by Wendt [46].

In Fig. 9 we show more detail of the torque evolution for the specific case of $\mathcal{R}=1.5$. In addition, at a number of discrete times, especially at local minima and maxima of the time-evolving torque curve, we show contour plots of the circumferential velocity $v_{\phi} / R_{\mathrm{i}} \Omega_{\mathrm{i}}$ along with the streamlines of 


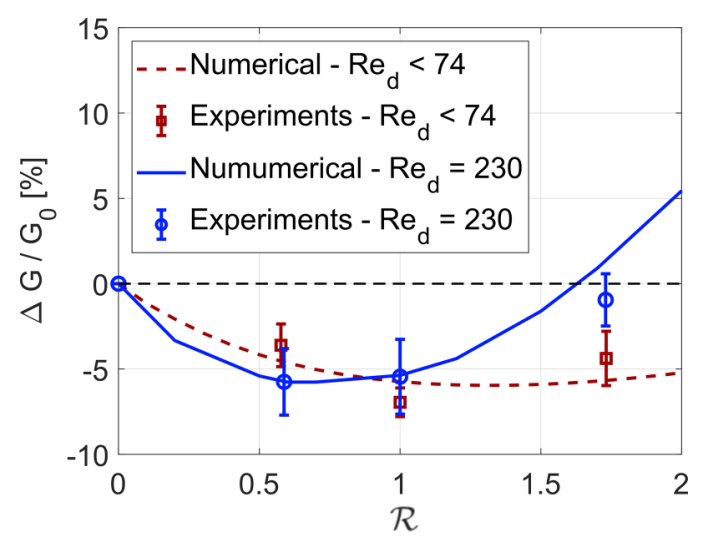

FIG. 10. Frictional torque change in the Taylor vortex regime with riblets of $\lambda / d=0.24$ at $\operatorname{Re}_{d}=230$ over a range of aspect ratios. The results of the torque change for the laminar Taylor-Couette flow for $\lambda / d=0.24$ as a function of the $\mathcal{R}$ are also shown here for comparison (dashed line and squares).

the secondary flow in the $(r, z)$ plate, indicating the structure of the axial and radial velocity fields. Initially (point $A$ ), inside each groove, small vortex pairs (arising from the two-dimensional nature of the flow inside the riblets) are created, while toward the outer cylinder larger vortex structures start to form. As time progresses $\left(t / t_{d} \approx 0.08\right.$, point $\left.B\right)$, the four pairs of Taylor vortices strengthen and start to interact with the smaller vortices inside the grooves. Later at $t / t_{d} \approx 0.15$ (corresponding to points $C$ and $D$ ), the larger Taylor vortices take over the smaller vortices inside the grooves and then the four vortex pairs take full form. Subsequently, a sudden jump in the torque is observed, as the vortex pairs interact and mix with each other to merge into the three pairs seen at the local torque maximum indicated by point $E$. At the next local minimum in the torque at point $F$, cell merging continues and the vortex pairs are down to two, but some remnants of older vortical structures are seen. Ultimately, when the flow reaches steady state $\left(t>t_{d}\right.$, point $\left.G\right)$, only two pairs of vortices are visible in the entire cell with $\Gamma=3.4$ and the torque subsequently remains constant in time.

Our experimental results in the Taylor vortex regime presented in Fig. 4 also showed that the total torque reduction measured on each $3 \mathrm{D}$ printed rotor was a function of the aspect ratio of the riblets, and similar nonmonotonic behavior is observed in the steady-state torque change as a function of the aspect ratio. A comparison of the computed and experimental changes on the torque for $\operatorname{Re}_{d}=230$, $\eta=0.63$, and riblets of wavelength $\lambda / d=0.24$ as a function of the aspect ratio $\mathcal{R}$ is shown in Fig. 10. At $\operatorname{Re}_{d}=230$ we are well above the transition from the CF to the TVF regime but still well below transition to the WVF regime. The results show a nonmonotonic behavior for the torque reduction as a function of the aspect ratio with a local minimum appearing between aspect ratios of 0.5 and unity. For comparison we also show the results of the torque reduction observed in the laminar flow for $\operatorname{Re}_{d}<74$ with the same riblet geometry, over the same range of aspect ratios.

Comparing the drag reduction achieved in the TVF $\left(\right.$ at $\left.\operatorname{Re}_{d}=230\right)$ compared with the Couette flow regime (at $\operatorname{Re}_{d} \leqslant 74$ ), it is apparent that in the Taylor vortex regime, friction reduction can only be achieved over a smaller range of aspect ratios, compared with the broad drag-reducing regime obtained in the homogeneous $\mathrm{CF}$ case. The maximum percentage reduction is quite similar between the two cases; however, this local minimum in the torque happens at a lower aspect ratio $(\mathcal{R} \approx 0.6-0.7)$ for the TVF, compared with the CF $(\mathcal{R} \approx 1.2-1.3)$.

This trend in the torque change can again be understood by considering in detail the local distribution of the traction on the riblet wall. As we discussed in Sec. IV A and in Eq. (13), the circumferential component of the traction vector is dependent on both $\tau_{\mathrm{rz}}$ and $\tau_{\mathrm{r} \phi}$ as well as the orientation of the normal vector to the wall. Here, with the Taylor vortices present, instead of plotting the traction distribution for only one riblet, in Fig. 11(a) we show the distribution of wall shear stress 


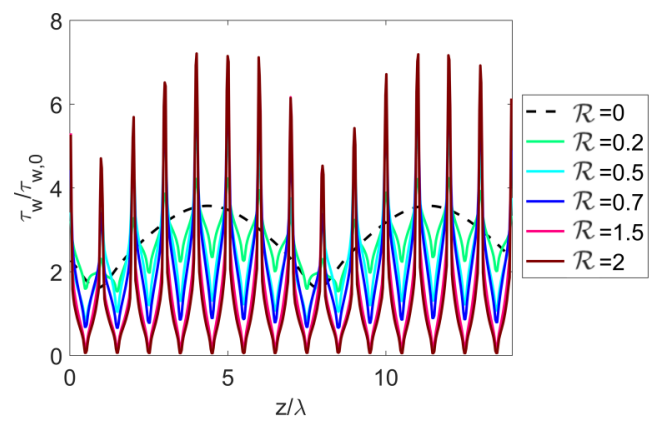

(a)

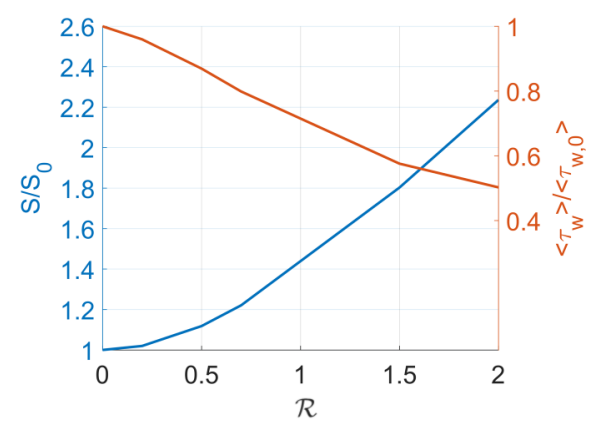

(b)

FIG. 11. (a) Distribution of the circumferential component of the viscous surface traction on the steady, rotating riblet-covered rotor wall in the TVF regime with riblet-covered inner cylinders. The shear stress is plotted for two Taylor vortex pair wavelengths $14 \lambda$ and for various aspect ratios $0 \leqslant \mathcal{R} \leqslant 2$ at $\operatorname{Re}_{d}=230$. The shear stress is nondimensionalized with the shear stress in the laminar Taylor-Couette flow with smooth rotors $\tau_{\mathrm{w}, 0}$ [defined in Eq. (2)]. (b) Evolution in average circumferential wall traction in Taylor vortex flow over riblet-covered rotors compared with the average shear stress on a smooth rotor as a function of the aspect ratio for the case of $\lambda / d=0.24$ and $\operatorname{Re}_{d}=230$ (red line) as well as the change in the wetted area $S / S_{0}$ as a function of $\mathcal{R}$ (blue line).

with axial distance over a length corresponding to two Taylor vortex pairs $(z / \lambda=14)$ in order to capture all of the details of the profiles. Here the traction is normalized by the traction distribution on a smooth wall in the case of the homogeneous CF conditions as shown in Eq. (2).

Compared to the CF regime, the two-dimensional nature of the velocity field in the TVF regime results in the inner rotor experiencing a nonuniform distribution in the local shear stress component $\tau_{\mathrm{r} \phi}$ even in the smooth rotor case $(\mathcal{R}=0)$ and this results in a nonuniform traction $\tau_{\mathrm{w}, \phi}$ at the wall. The additional presence of the riblets further changes the traction distribution such that the traction experienced inside the grooves is less than the traction close to the peaks of the riblets. On the other hand, arising from the vortical modulation of the velocity profiles in the axial direction, the traction distribution is not the same for each and every riblet; therefore, the maxima and minima of the curves follow the overall shape of the shear stress distribution arising due to the Taylor vortices (as in the case of $\mathcal{R}=0$ ) while also possessing local frequency maxima and minima at a higher spatial frequency due to the presence of the riblets. This shows that both the riblets on the rotor surface and the Taylor vortices that develop in the flow field within the gap play essential roles in the net changes seen in the frictional torque.

Due to the distribution of the traction on riblet-covered rotors, we notice that a substantial portion of the area of each riblet experiences a traction lower than the corresponding modulated traction on the smooth surface. Increasing the aspect ratio results in a larger and larger fraction of the rotor experiencing a lower shear stress inside the grooves. For the deepest grooves $(\mathcal{R}=1.5$ and 2$)$ the flow moves with almost the same velocity as the rotating wall at the very bottom of the groove. Consequently, as we show in Fig. 11(b), the average shear stress distribution (averaged over one wavelength of the vortex pair) in TVF over the riblets decreases as the aspect ratio of the riblets increase. However, due to the Taylor vortices, the peaks of the riblets also experience a modulated increase in the shear stress compared to the case of the homogeneous CF regime. Especially in higher- $\mathcal{R}$ cases, the local minima of the shear stress profiles remain rather constant along the length of the cylinder while the local stress maxima change along each axial period following the longwavelength distribution in the traction arising as a result of the Taylor vortices. The average traction distribution over one complete period of the vortex pairs $(z / \lambda=7)$ as a function of the aspect ratio of the riblets is presented in Fig. 11(b). Similar to the CF case [see Fig. 7(b)], the average traction decreases monotonically with aspect ratio whereas the total wetted area increases. Therefore, the net torque reduction which arises from the interplay of the reduction in the average traction, coupled 
with the increase in the wetted area of the grooved rotor, once again varies nonmonotonically and goes through a local minimum as a function of the aspect ratio of the riblets. However, it should be noted that due to the superimposed axial modulation in the shear stress experienced on the peaks of the riblets, the decrease in the average traction seen in Fig. 11(b) is not as substantial as in the axisymmetric Couette flow regime. Contrasting Figs. 11(b) and 7(b), it can be seen that the reduction in the average traction in the presence of Taylor vortices only overcomes the geometric effect of the area increase for a smaller range of aspect ratio. Hence the regime corresponding to a net torque reduction can only be achieved for a smaller range of aspect ratios compared with the laminar case. As shown in Fig. 10, our experimental measurements in the TVF regime agree very well with the results of the numerical simulations presented here.

\section{CONCLUSION}

In this paper we have used the Taylor-Couette geometry to study the drag-reducing effect of riblets on a steady torsional flow and to measure the net torque reduction they can offer over a range of flow speeds. Taylor-Couette flow is a canonical flow with a well-defined geometry and does not have the additional complications of the entrance or exit length effects encountered in pipe or channel flow experiments. The experimental setup was designed and built using several complementary manufacturing methods. The inner cylinders were 3D printed using a Form $1+3 \mathrm{D}$ printer with various $\mathrm{V}$-groove riblet sizes varying in both aspect ratio and wavelength. The fixed outer cylinder was constructed using a CNC mill and the setup was mounted on a stress-controlled rheometer to allow for accurate measurement of the angular velocity and frictional viscous torque on the inner cylinder.

To understand the results of the experiments, axisymmetric and periodic numerical simulations were performed on the homogeneous purely azimuthal Couette flow with riblet surfaces and the results were compared with experimental results. The experimental and numerical results confirm that using riblet surfaces confers an ability to reduce the frictional torque exerted by the fluid on the rotor. Using an asymptotic expansion of the velocity profile, along with a domain perturbation analysis, we also present a theoretical prediction for the changes in torque expected in the limit of shallow riblets. In the limit of $\mathcal{R} \rightarrow 1$, this theoretical model captures the results very well; however, it fails to capture the nonmonotonic dependence of the torque change with aspect ratio observed for larger $\mathcal{R}$.

The above discussions all focused on the laminar flow regime where the effect of changes in the velocity ( or $\mathrm{Re}_{d}$ ) on either the smooth case or the riblet-covered rotors is linear and thus drops out of the problem when ratios such as $\Delta G / G_{0}$ are calculated. However, we have also investigated the case of higher Reynolds number $\left(\operatorname{Re}_{d}=230\right)$ in the Taylor vortex regime with $\eta=0.63$ and $\Gamma=3.4$ and studied in detail the effect of the riblet-covered rotors on the changes in the frictional torque on the inner cylinder. Time-dependent numerical simulations of the flow over riblets with $\lambda / d=0.24$ and aspect ratios in the range of $0 \leqslant \mathcal{R} \leqslant 2$ were analyzed to understand the interactions of the riblets with the Taylor vortices.

Computations showed that the riblet peaks are directly in contact with the Taylor vortices that develop at steady state. These vortical structures try to penetrate inside the grooves and hence the riblet peaks are in contact with high-momentum fluid and experience a higher shear stress compared to the rest of the groove surfaces. The resulting integrated changes in torque were calculated as a function of the aspect ratio of the grooves and compared with the corresponding laminar situation. Similar to the laminar case, the riblets reduce the average shear stress distribution over the textured rotor as the aspect ratio of the riblets is increased. However, the presence of the Taylor vortices and their interactions with the riblets result in an additional increase in the total frictional torque and hence the reduction in the average shear stress distribution is smaller than the decrease observed in the laminar axisymmetric case. The nonmonotonic trends observed in the torque change as a function of $\mathcal{R}$ are extended into the Taylor vortex regime; however, both the computations and 
experiments show that a smaller range of aspect ratios are able to provide a reduction in the total frictional drag force at $\operatorname{Re}_{d}=230$.

Given the complexity of the Taylor vortex flow and the various bifurcations that are possible for this flow, it is intriguing to consider additional cases with different riblet geometries and other Reynolds numbers. Future work should focus on analysis of inertial effects on the torque changes throughout the Taylor vortex flow regime. In addition, fully 3D simulations for larger Reynolds numbers should be performed for the full circumferential extent of the concentric cylinders geometry, since the ensuing flow states are not fully axisymmetric. However, such simulations are expensive and would require substantially larger computational resources.

\section{APPENDIX A: THEORETICAL MODEL FOR CHANGES IN TORQUE USING ASYMPTOTIC EXPANSION AND DOMAIN PERTURBATION OF RIBLET TEXTURED ROTORS AT SMALL ASPECT RATIOS}

To understand the effect of the size of the riblets, we can expand the theoretical calculations of the cylindrical Couette flow to include higher-order terms for small riblets with amplitudes much smaller than the radius of the inner cylinder $\left(A \ll R_{\mathrm{i}}\right)$. To consider this small parameter and be able to compare with the rest of the dimensionless groups introduced throughout this Appendix, we can write

$$
\frac{A}{R_{\mathrm{i}}}=\frac{\mathcal{R}}{2} \frac{\lambda}{R_{\mathrm{i}}}=\frac{\mathcal{R}}{2} \varepsilon
$$

and hence we can think of two small parameters in the problem. One is the aspect ratio of the riblets $\mathcal{R}=2 A / \lambda$ (meaning very shallow riblets) as well $\varepsilon=\lambda / R_{\mathrm{i}}$, where at the limit of $A / R_{\mathrm{i}} \rightarrow 0$, the results of the torque calculations reduce to the results from a smooth cylinder in steady TaylorCouette flow and at a constant aspect ratio increasing the wavelength would result in an increase in $\varepsilon$.

We then assume that the azimuthal velocity $v_{\phi}$ profile in the presence of riblets can be written in the form below, which has a zeroth-order and a first-order term, with the zeroth order being the same profile as the solution for smooth laminar Couette setup. Any effect of higher-order terms is neglected,

$$
v_{\phi}=v_{\phi}^{(0)}+\mathcal{R} \varepsilon v_{\phi}^{(1)}+O\left(\mathcal{R}^{2} \varepsilon^{2}\right)+\cdots,
$$

and the zeroth-order Couette solution is only a function of the radial direction $r$ :

$$
v_{\phi}^{(0)}=\frac{\eta}{1-\eta^{2}}\left(\frac{R_{\mathrm{o}}}{r}-\frac{r}{R_{\mathrm{o}}}\right) R_{\mathrm{i}} \Omega_{\mathrm{i}} .
$$

Also, on the basis of asymptotic analysis and computations we assume that $v_{\mathrm{r}}$ is of second and higher order and thus does not appear in the present analysis whereas $v_{z}$ is of first order and is only a function of the radial direction. More details justifying this assumption can be found at the end of this Appendix. Therefore, we write

$$
\left[\begin{array}{c}
v_{\mathrm{r}} \\
v_{\phi}(r) \\
v_{\mathrm{z}}
\end{array}\right]=\left[\begin{array}{c}
0 \\
v_{\phi}^{(0)} \\
0
\end{array}\right]+\mathcal{R} \varepsilon\left[\begin{array}{c}
0 \\
v_{\phi}^{(1)}(r, z) \\
v_{\mathrm{z}}^{(1)}(r)
\end{array}\right]+O\left(\mathcal{R}^{2} \varepsilon^{2}\right)+\cdots
$$

Thus, the radial and axial velocities do not enter the azimuthal component of the equations of motion and can be written as

$$
\frac{\partial}{\partial r}\left(\frac{1}{r} \frac{\partial}{\partial r}\left(r v_{\phi}\right)\right)+\frac{\partial^{2} v_{\phi}}{\partial z^{2}}=0
$$


After substituting for $v_{\phi}$ from Eq. (A2) (neglecting the second- and higher-order terms), this can be divided into two separate equations

$$
\begin{gathered}
\frac{\partial}{\partial r}\left(\frac{1}{r} \frac{\partial}{\partial r}\left[r\left(v_{\phi}^{(0)}\right)\right]\right)=0 \\
\frac{\partial}{\partial r}\left(\frac{1}{r} \frac{\partial}{\partial r}\left[r\left(v_{\phi}^{(1)}\right)\right]\right)+\frac{\partial^{2}}{\partial z^{2}}\left(v_{\phi}^{(1)}\right)=0 .
\end{gathered}
$$

Since Eq. (A3) satisfies the equations of motion for Taylor-Couette flow, in a smooth-walled cylinder then Eq. (A6) is already satisfied and thus the equation of motion for the first-order term simplifies to Eq. (A7).

Now to solve this equation we make some simplifying assumptions. First we note that the boundary condition on the inner cylinder is a constant rotation rate, given by $\Omega_{\mathrm{i}}$. Therefore, the velocity boundary condition on the riblets is not uniform and is dependent on the local position on the riblets wall. If $r_{\mathrm{w}}(z)$ is the local radial location on the riblet inner cylinder, then the boundary condition for $v_{\phi}$ is written as (note the symmetry about the line of $z=\lambda / 2$ )

$$
\begin{gathered}
v_{\phi, \mathrm{BC}}=r_{\mathrm{w}} \Omega_{\mathrm{i}}=\left(R_{\mathrm{i}}-\mathcal{R} z\right) \Omega_{\mathrm{i}} \quad \text { for } 0 \leqslant z \leqslant \frac{\lambda}{2}, \\
v_{\phi, \mathrm{BC}}=r_{\mathrm{w}} \Omega_{\mathrm{i}}=\left[R_{\mathrm{i}}-\mathcal{R}(\lambda-z)\right] \Omega_{\mathrm{i}} \quad \text { for } \frac{\lambda}{2} \leqslant z \leqslant \lambda .
\end{gathered}
$$

Thus, for the first-order term in azimuthal velocity, the boundary condition for $0 \leqslant z \leqslant \lambda / 2$ can be written in nondimensional form as

$$
\mathcal{R} \varepsilon \frac{v_{\phi, \mathrm{BC}}^{(1)}}{R_{\mathrm{i}} \Omega_{\mathrm{i}}}=\left(1-\mathcal{R} \frac{z}{R_{\mathrm{i}}}\right)-\left.\frac{1}{R_{\mathrm{i}} \Omega_{\mathrm{i}}} v_{\phi}^{(0)}\right|_{r=R_{\mathrm{i}}-\mathcal{R} z} .
$$

Substituting for the zeroth-order term from Eq. (A3) at $r=R_{\mathrm{i}}$, we have (by replacing $R_{\mathrm{i}} / R_{\mathrm{o}}$ with $\eta$ and $\left.\mathcal{R} z / R_{\mathrm{i}}=\mathcal{R} \varepsilon z / \lambda\right)$

$$
\mathcal{R} \varepsilon \frac{v_{\phi, \mathrm{BC}}^{(1)}}{R_{\mathrm{i}} \Omega_{\mathrm{i}}}=\left(1-\mathcal{R} \varepsilon \frac{z}{\lambda}\right)-\frac{1}{1-\eta^{2}} \frac{1}{1-\mathcal{R} \varepsilon \frac{z}{\lambda}}+\frac{\eta^{2}}{1-\eta^{2}}\left(1-\mathcal{R} \varepsilon \frac{z}{\lambda}\right) .
$$

So for shallow riblets with amplitude much smaller than $R_{\mathrm{i}}$, i.e., $\varepsilon \mathcal{R} \ll 1$, we can use the Taylor series expansion

$$
\frac{1}{1-\mathcal{R} \varepsilon \frac{z}{\lambda}}=1+\mathcal{R} \varepsilon \frac{z}{\lambda}+O\left(\mathcal{R}^{2} \varepsilon^{2}\right)+\cdots .
$$

Thus the boundary condition (neglecting the second- and higher-order terms) can be simplified to

$$
\frac{v_{\phi, \mathrm{BC}}^{(1)}}{R_{\mathrm{i}} \Omega_{\mathrm{i}}}=\frac{-2}{1-\eta^{2}} \frac{z}{\lambda}+O(\mathcal{R} \varepsilon)+\cdots .
$$

In the limit of shallow grooves and small $\varepsilon$, Eq. (A7) can be solved analytically with boundary conditions of $\Omega_{\mathrm{o}}=0$ at $r=R_{\mathrm{o}}$ and Eq. (A13) at $r=R_{\mathrm{i}}$. The solution can be written as

$$
\frac{v_{\phi}^{(1)}}{R_{\mathrm{i}} \Omega_{\mathrm{i}}}=\frac{-2 \eta}{\left(1-\eta^{2}\right)^{2}}\left(\frac{R_{\mathrm{o}}}{r}-\frac{r}{R_{\mathrm{o}}}\right) \frac{z}{\lambda} .
$$

Now, similarly to find the change in the torque due to the geometry, the torque can also be expanded in terms of a zeroth- and first-order term as

$$
T=T^{(0)}+\mathcal{R} \varepsilon T^{(1)}+O\left(\mathcal{R}^{2} \varepsilon^{2}\right)+\cdots,
$$

where the torque on a smooth rotor $\left(T^{(0)}\right)$ is given by Eq. (4). Thus, to calculate the first-order term in the torque, we use the first-order term in velocity to calculate the additional contribution to the 


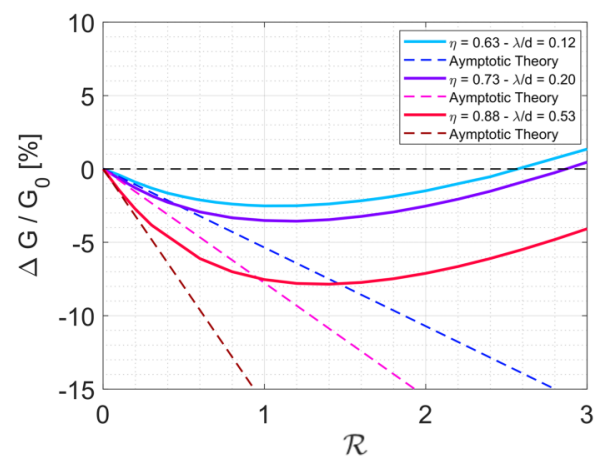

(a)

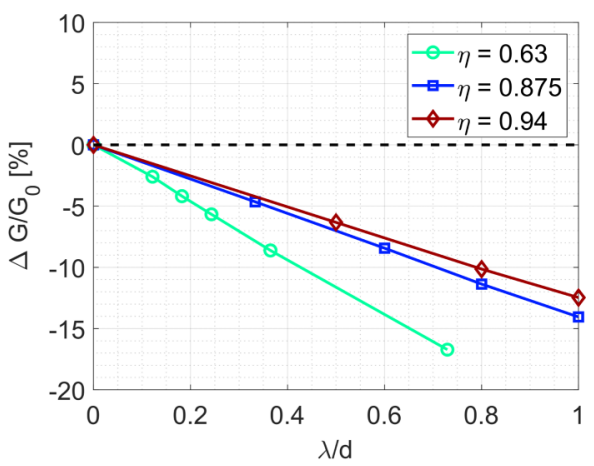

(b)

FIG. 12. (a) Torque-reduction results from numerical simulation of Taylor-Couette flow with riblet-covered rotors having $\lambda / R_{\mathrm{i}}=0.07$ and for different radius ratios $\eta$ and $\lambda / d$ as a function of the aspect ratio $\mathcal{R}$. Theoretical results from Eq. (11) for shallow riblets are also shown in the figure with dashed lines. (b) Torque reduction as a function of the wavelength of the riblets at a fixed value of the aspect ratio $\mathcal{R}=1.00$.

shear rate on the wall. Since we are assuming $\mathcal{R} \ll 1$ we can approximate the shear rate on the riblet wall as the shear rate calculated at $r=R_{\mathrm{i}}$. Thus

$$
\dot{\gamma}_{\mathrm{r} \phi}^{(1)}=r \frac{\partial}{\partial r}\left(\frac{v_{\phi}^{(1)}}{r}\right)
$$

and then the shear stress on $r=R_{\mathrm{i}}$ can be written as

$$
\tau_{\mathrm{r} \phi}^{(1)}=\mu \frac{4 \eta}{\left(1-\eta^{2}\right)^{2}} \frac{R_{\mathrm{o}}}{R_{\mathrm{i}}^{2}} \frac{z}{\lambda} R_{\mathrm{i}} \Omega_{\mathrm{i}}
$$

Hence the first-order correction term in torque can be calculated and simplified as

$$
T^{(1)}=2 \int_{0}^{\lambda / 2} \tau_{\mathrm{r} \phi}^{(1)} 2 \pi R_{\mathrm{i}}^{2} d z=\frac{1}{2}\left(4 \pi \rho v^{2} \lambda\right) \frac{1}{\left(1-\eta^{2}\right)^{2}} \frac{R_{\mathrm{i}}}{d} \operatorname{Re}_{d}
$$

and in dimensionless form

$$
G^{(1)}=\frac{T^{(1)}}{4 \pi \rho v^{2} \lambda}=\frac{1}{2} \frac{1}{\left(1-\eta^{2}\right)^{2}} \frac{R_{\mathrm{i}}}{d} \operatorname{Re}_{d} .
$$

Now to find the change in torque compared to a smooth rotor, we have

$$
\frac{\Delta G}{G_{0}}=-\frac{1}{2} \mathcal{R}\left(\frac{\lambda}{d}\right)\left(\frac{1}{\eta(1+\eta)}\right) .
$$

This equation predicts that the changes in torque are also a function of the size of the riblets $\lambda / d$ as well as the geometry of the specific Taylor-Couette cell [from the term $1 / \eta(1+\eta)$ ]. The computational results presented above were generated by keeping the radius ratio $\eta$ and the gap size $d$ constant and only changing the wavelength of the riblets. Figure 12(a) shows the results of numerical simulation where the wavelength of the riblets and the radius of the inner cylinder were kept constant at $\lambda / R_{\mathrm{i}}=0.07$ and the radius of the outer cylinder and the gap were changed and therefore both $\eta$ and $\lambda / d$ were changed. The results presented here also show a nonmonotonic relationship similar to the effect of varying aspect ratio on the reduction in torque, but it can be seen that the asymptotic theory once again can capture the slope of the curves at $\mathcal{R} \rightarrow 0$. Thus the theory and supporting experimental and numerical data show that the torque reduction is initially directly 
dependent on the size of the riblets with respect to the size of the Taylor-Couette geometry (i.e., $A / R_{\mathrm{i}}$ ), as well as the radius ratio $\eta$ of the Taylor-Couette cell used.

On the other hand, when the aspect ratio of the riblets is kept constant, the torque reduction achieved is directly dependent on the wavelength of the riblets and as the wavelength $\lambda / d$ increases, the torque reduction achieved is enhanced as seen in Figs. 4 and 5. If the torque reduction at a constant $\mathcal{R}$ is plotted as a function of the wavelength $\lambda / d$ [shown in Fig. 12(b)], it can be seen that the extent of the torque reduction achieved follows a linear trend with increasing wavelength. The torque reduction also depends on the curvature of the base flow and steadily decreases as $\eta \rightarrow 1$. Finally, it should be noted that since the asymptotic theory relies on an expansion about a chosen radius (here $R_{\mathrm{i}}$ ), the theory will predict the same percentage change in circumferential force and torque and thus overestimates the reduction in force as compared to the calculations presented in Fig. 5(d).

Now, to support the earlier ansatz [i.e., $v_{\mathrm{r}}^{(1)}=0$ in Eq. (A4)], we attempt to solve the equations of motion for steady cylindrical Couette flow over V-groove riblet surfaces. We start by decomposing the solution for the velocity and pressure distribution as a base solution and a first-order perturbation for small riblets with the earlier assumption that $\mathcal{R} \varepsilon \ll 1$. Here the base flow is the cylindrical Couette flow over smooth rotors discussed earlier and the perturbation term is due to the presence of the riblets. Therefore, we can expand the pressure and velocity profiles in the forms

$$
\begin{aligned}
& p=p^{(0)}+\mathcal{R} \varepsilon p^{(1)}+O\left(\mathcal{R}^{2} \varepsilon^{2}\right)+\cdots, \\
& \boldsymbol{v}=\left[\begin{array}{c}
v_{\mathrm{r}} \\
v_{\phi} \\
v_{\mathrm{z}}
\end{array}\right]=\left[\begin{array}{c}
0 \\
v_{\phi}^{(0)} \\
0
\end{array}\right]+\mathcal{R} \varepsilon\left[\begin{array}{c}
v_{\mathrm{r}}^{(1)} \\
v_{\phi}^{(1)} \\
v_{\mathrm{z}}^{(1)}
\end{array}\right]+O\left(\mathcal{R}^{2} \varepsilon^{2}\right)+\cdots
\end{aligned}
$$

First the component of the steady axisymmetric Navier-Stokes equation in the $\phi$ direction is written as

$$
\rho\left(\frac{v_{\mathrm{r}}}{r} \frac{\partial v_{\phi}}{\partial r}+v_{\mathrm{z}} \frac{\partial v_{\phi}}{\partial z}\right)=\mu\left[\frac{\partial}{\partial r}\left(\frac{1}{r} \frac{\partial}{\partial r}\left(r v_{\phi}\right)\right)+\frac{\partial^{2} v_{\phi}}{\partial z^{2}}\right] .
$$

With the no-slip boundary condition on the inner and outer cylinders, i.e., $v_{\mathrm{r}}^{(1)}\left(r=R_{\mathrm{i}}\right)=v_{\mathrm{r}}^{(1)}(r=$ $R_{\mathrm{o}}$ ) $=0$, it is assumed that $v_{\mathrm{r}}^{(1)}=0$. Thus, substituting the expansion (A22) for the velocity and gathering the first-order terms with the assumption that $v_{\mathrm{r}}^{(1)}=0$ gave us Eq. (A7), which was solved subject to the boundary condition given by (A13) and the solution is presented in Eq. (A14). Now using the axisymmetric Navier-Stokes equation in the radial direction

$$
\rho\left(v_{\mathrm{r}} \frac{\partial v_{\mathrm{r}}}{\partial r}+v_{\mathrm{z}} \frac{\partial v_{\mathrm{r}}}{\partial z}-\frac{v_{\phi}^{2}}{r}\right)=\frac{\partial p}{\partial r}+\mu\left[\frac{\partial}{\partial r}\left(\frac{1}{r} \frac{\partial}{\partial r}\left(r v_{\mathrm{r}}\right)\right)+\frac{\partial^{2} v_{\mathrm{r}}}{\partial z^{2}}\right]
$$

and again substituting the expansion and gathering the first-order terms, we obtain

$$
\rho \frac{2 v_{\phi}^{(0)} v_{\phi}^{(1)}}{r}=\frac{\partial p^{(1)}}{\partial r}
$$

and thus

$$
\frac{\partial p^{(1)}}{\partial r}=\rho \frac{2 \eta^{2}}{\left(1-\eta^{2}\right)^{3}} \frac{1}{r}\left(\frac{R_{\mathrm{o}}}{r}-\frac{r}{R_{\mathrm{o}}}\right)^{2} \frac{z}{\lambda}\left(R_{\mathrm{i}} \Omega_{\mathrm{i}}\right)^{2} .
$$

Integrating this equation, one obtains an equation for the pressure distribution as

$$
p^{(1)}=\frac{2 \eta^{2}}{\left(1-\eta^{2}\right)^{3}} \frac{z}{\lambda}\left[-\frac{1}{2}\left(\frac{R_{\mathrm{o}}}{r}\right)^{2}-2 \ln \left(\frac{r}{R_{\mathrm{o}}}\right)+\frac{1}{2}\left(\frac{r}{R_{\mathrm{o}}}\right)^{2}\right] \rho\left(R_{\mathrm{i}} \Omega_{\mathrm{i}}\right)^{2} .
$$


Therefore, the pressure gradient in the $z$ direction is written as

$$
\frac{\partial p^{(1)}}{\partial z}=\frac{2 \eta^{2}}{\left(1-\eta^{2}\right)^{3}} \frac{1}{\lambda}\left[-\frac{1}{2}\left(\frac{R_{\mathrm{o}}}{r}\right)^{2}-2 \ln \left(\frac{r}{R_{\mathrm{o}}}\right)+\frac{1}{2}\left(\frac{r}{R_{\mathrm{o}}}\right)^{2}\right] \rho\left(R_{\mathrm{i}} \Omega_{\mathrm{i}}\right)^{2},
$$

which is only a function of the radial direction $r$. To simplify, we denote the right-hand side of Eq. (A28) by $f(r)$ and use the component of the steady axisymmetric Navier-Stokes equation in the axial direction

$$
\rho\left(v_{\mathrm{r}} \frac{\partial v_{\mathrm{z}}}{\partial r}+v_{\mathrm{z}} \frac{\partial v_{\mathrm{z}}}{\partial z}\right)=-\frac{\partial p}{\partial z}+\mu\left[\frac{1}{r} \frac{\partial}{\partial r}\left(r \frac{\partial v_{\mathrm{z}}}{\partial r}\right)+\frac{\partial^{2} v_{\mathrm{z}}}{\partial z^{2}}\right] .
$$

Substituting for the pressure and velocity expansion and collecting the first-order terms simplifies Eq. (A29) to

$$
\mu\left[\frac{1}{r} \frac{\partial}{\partial r}\left(r \frac{\partial v_{\mathrm{z}}^{(1)}}{\partial r}\right)+\frac{\partial^{2} v_{\mathrm{z}}^{(1)}}{\partial z^{2}}\right]=\frac{\partial p^{(1)}}{\partial z}=f(r) .
$$

Therefore, the solution to $v_{\mathrm{z}}$ has the form

$$
v_{\mathrm{z}}^{(1)}=g(r)+C_{1} z+D_{1}
$$

and with the boundary condition

$$
\left.\frac{\partial v_{\mathrm{z}}^{(1)}}{\partial z}\right|_{z=0}=-\left.\frac{\partial v_{\mathrm{z}}^{(1)}}{\partial z}\right|_{z=\lambda / 2}
$$

one thus finds that $C_{1}=0$. Therefore, using the continuity equation (at this order)

$$
\frac{1}{r} \frac{\partial}{\partial r}\left(r v_{\mathrm{r}}^{(1)}\right)+\frac{\partial v_{\mathrm{z}}^{(1)}}{\partial z}=0
$$

and substituting the above form for the first-order terms, one gets

$$
\frac{1}{r} \frac{\partial}{\partial r}\left(r v_{\mathrm{r}}^{(1)}\right)=0
$$

which can be solved to give

$$
v_{\mathrm{r}}^{(1)}=\frac{D_{1}}{r}
$$

and with the no-slip boundary condition at the inner and outer walls, $D_{1}=0$ and thus $v_{\mathrm{r}}^{(1)}=0$, confirming the assumption that was made earlier to solve the equations. Any radial or recirculating velocity that is induced by the riblets thus develops only at higher orders $O\left(\varepsilon^{2} \mathcal{R}^{2}\right)$ and beyond.

For $g(r)$, substituting $v_{\mathrm{z}}^{(1)}$ into Eq. (A30) and integrating twice we have

$$
g(r)=\int\left(\frac{1}{r} \int \frac{r}{\mu} f(r) d r+\frac{C_{2}}{r}\right) d r+D_{2}
$$

subject to no-slip boundary conditions at $r=R_{\mathrm{i}}$ and $r=R_{\mathrm{0}}$. Thus the velocity field at first order is $v_{\mathrm{r}}^{(1)}=0$ and $v_{\mathrm{z}}^{(1)}=g(r)$, where

$$
g(r)=K\left[\left(\frac{r}{R_{\mathrm{o}}}\right)^{4}+16\left(\frac{r}{R_{\mathrm{o}}}\right)^{2}-16\left(\frac{r}{R_{\mathrm{o}}}\right)^{2} \ln \left(\frac{r}{R_{\mathrm{o}}}\right)-8 \ln \left(\frac{r}{R_{\mathrm{o}}}\right)^{2}\right]+C_{2} \ln \left(\frac{r}{R_{\mathrm{o}}}\right)+D_{2},
$$


with

$$
\begin{aligned}
K & =\frac{\rho}{\mu} \frac{2 \eta^{2}}{\left(1-\eta^{2}\right)^{3}} \frac{R_{\mathrm{o}}^{2}}{32 \lambda}\left(R_{\mathrm{i}} \Omega_{\mathrm{i}}\right)^{2}, \\
C_{2} & =\frac{17-\eta^{4}-16 \eta^{2}+16 \eta^{2} \ln (\eta)+8 \ln (\eta)^{2}}{\ln (\eta)} K,
\end{aligned}
$$

and

$$
D_{2}=-17 K
$$

[1] M. J. Walsh, Riblets, in Viscous Drag Reduction in Boundary Layers, edited by D. M. Bushnell and J. N. Hefner, Progress in Astronautics and Aeronautics Vol. 123 (AIAA, Washington, DC, 1990), pp. 203-261.

[2] M. J. Walsh, Riblets as a viscous drag reduction technique, AIAA J. 21, 485 (1983).

[3] M. J. Walsh and A. M. Lindemann, Proceedings of the 22nd Aerospace Sciences Meeting, Reno, 1984 (AIAA, Reston, 1984), p. 347.

[4] D. Hooshmand, R. Youngs, J. M. Wallace, and J. L. Balint, Proceedings of the AlAA 21st Aerospace Sciences Meeting, Reno, 1983 (AIAA, Reston, 1983), paper 83-0230.

[5] S.-i. Nakao, Application of V shape riblets to pipe flows, J. Fluids Eng. 113, 587 (1991).

[6] L. Djenidi, L. C. Squire, and A. M. Savill, in Recent Developments in Turbulence Management, edited by K.-S. Choi (Springer, Dordrecht, 1991), pp. 65-92.

[7] L. Djenidi, F. Anselmet, J. Liandrat, and L. Fulachier, Laminar boundary layer over riblets, Phys. Fluids 6, 2993 (1994).

[8] D. C. Chu and G. E. Karniadakis, A direct numerical simulation of laminar and turbulent flow over riblet-mounted surfaces, J. Fluid Mech. 250, 1 (1993).

[9] H. Choi, P. Moin, and J. Kim, On the effect of riblets in fully developed laminar channel flows, Phys. Fluids A 3, 1892 (1991).

[10] H. Choi, P. Moin, and J. Kim, Direct numerical simulation of turbulent flow over riblets, J. Fluid Mech. 255, 503 (1993).

[11] S. F. Tardu, Coherent structures and riblets, Appl. Sci. Res. 54, 349 (1995).

[12] S. Raayai-Ardakani and G. H. McKinley, Drag reduction using wrinkled surfaces in high Reynolds number laminar boundary layer flows, Phys. Fluids 29, 093605 (2017).

[13] S. Raayai-Ardakani and G. H. McKinley, Geometric optimization of riblet-textured surfaces for drag reduction in laminar boundary layer flows, Phys. Fluids 31, 053601 (2019).

[14] S. Raayai Ardakani, Geometry mediated drag reduction using riblets and wrinkled surface textures, Ph.D. thesis, Massachusetts Institute of Technology, 2018.

[15] S. Grossmann, D. Lohse, and C. Sun, High-Reynolds number Taylor-Couette turbulence, Annu. Rev. Fluid Mech. 48, 53 (2016).

[16] K. Avila and B. Hof, High-precision Taylor-Couette experiment to study subcritical transitions and the role of boundary conditions and size effects, Rev. Sci. Instrum. 84, 065106 (2013).

[17] D. P. M. van Gils, G.-W. Bruggert, D. P. Lathrop, C. Sun, and D. Lohse, The Twente turbulent Taylor-Couette $\left(\mathrm{T}^{3} \mathrm{C}\right)$ facility: Strongly turbulent (multiphase) flow between two independently rotating cylinders, Rev. Sci. Instrum. 82, 025105 (2011).

[18] T. Hall and D. Joseph, Rotating cylinder drag balance with application to riblets, Exp. Fluids 29, 215 (2000).

[19] A. J. Greidanus, R. Delfos, S. Tokgoz, and J. Westerweel, Turbulent Taylor-Couette flow over riblets: Drag reduction and the effect of bulk fluid rotation, Exp. Fluids 56, 107 (2015).

[20] T. H. Van den Berg, S. Luther, D. P. Lathrop, and D. Lohse, Drag Reduction in Bubbly Taylor-Couette Turbulence, Phys. Rev. Lett. 94, 044501 (2005). 
[21] K. Sugiyama, E. Calzavarini, and D. Lohse, Microbubbly drag reduction in Taylor-Couette flow in the wavy vortex regime, J. Fluid Mech. 608, 21 (2008).

[22] B. J. Rosenberg, T. Van Buren, M. K. Fu, and A. J. Smits, Turbulent drag reduction over air-and liquidimpregnated surfaces, Phys. Fluids 28, 015103 (2016).

[23] D. Panchanathan, A. Rajappan, K. K. Varanasi, and G. H. McKinley, Plastron regeneration on submerged superhydrophobic surfaces using in situ gas generation by chemical reaction, ACS Appl. Mater. Interfaces 10, 33684 (2018).

[24] D. W. Bechert, M. Bruse, W. Hage, J. G. T. Van der Hoeven, and G. Hoppe, Experiments on drag-reducing surfaces and their optimization with an adjustable geometry, J. Fluid Mech. 338, 59 (1997).

[25] X. Zhu, R. Ostilla-Mónico, R. Verzicco, and D. Lohse, Direct numerical simulation of Taylor-Couette flow with grooved walls: Torque scaling and flow structure, J. Fluid Mech. 794, 746 (2016).

[26] C. D. Andereck, S. S. Liu, and H. L. Swinney, Flow regimes in a circular Couette system with independently rotating cylinders, J. Fluid Mech. 164, 155 (1986).

[27] P. H. Roberts, Appendix to experiments on the stability of viscous flow between rotating cylinders. VI. Finite-amplitude experiments, Proc. R. Soc. London Ser. A 238, 531 (1965).

[28] C. W. Macosko, Rheology: Principles, Measurements, and Applications (Wiley-VCH, Weinheim, 1994).

[29] R. J. Donnelly and K. W. Schwarz, Experiments on the stability of viscous flow between rotating cylinders. VI. Finite-amplitude experiments, Proc. R. Soc. London Ser. A 283, 531 (1965).

[30] A. Davey, R. C. Di Prima, and J. T. Stuart, On the instability of Taylor vortices, J. Fluid Mech. 31, 17 (1968).

[31] G. I. Taylor, Stability of a viscous liquid contained between two rotating cylinders, Philos. Trans. R. Soc. London 223, 289 (1923).

[32] D. P. Lathrop, J. Fineberg, and H. L. Swinney, Transition to shear-driven turbulence in Couette-Taylor flow, Phys. Rev. A 46, 6390 (1992).

[33] D. P. Lathrop, J. Fineberg, and H. L. Swinney, Turbulent Flow between Concentric Rotating Cylinders at Large Reynolds Number, Phys. Rev. Lett. 68, 1515 (1992).

[34] See Supplemental Material at http://link.aps.org/supplemental/10.1103/PhysRevFluids.5.124102 for additional figures and details as described in the main text.

[35] D. W. Bechert, M. Bruse, and W. Hage, Experiments with three-dimensional riblets as an idealized model of shark skin, Exp. Fluids 28, 403 (2000).

[36] M. Khan, Proceedings of the 4th Joint Fluid Mechanics, Plasma Dynamics and Lasers Conference, Atlanta, 1986 (AIAA, Reston, 1986).

[37] D. Coles, Transition in circular Couette flow, J. Fluid Mech. 21, 385 (1965).

[38] C. A. Jones, Nonlinear Taylor vortices and their stability, J. Fluid Mech. 102, 249 (1981).

[39] J. E. Burkhalter and E. L. Koschmieder, Steady supercritical Taylor vortices after sudden starts, Phys. Fluids 17, 1929 (1974).

[40] M. Gorman and H. L. Swinney, Spatial and temporal characteristics of modulated waves in the circular Couette system, J. Fluid Mech. 117, 123 (1982).

[41] T. B. Benjamin and T. Mullin, Anomalous modes in the Taylor experiment, Proc. R. Soc. London Ser. A 377, 221 (1981).

[42] C. L. Streett and M. Y. Hussaini, Finite Length Taylor Couette Flow, in Stability of Time Dependent and Spatially Varying Flows, edited by D. L. Dwoyer and M. Y. Hussaini (Springer, New York, 1987), pp. 312-334.

[43] T. Mullin and T. B. Benjamin, Transition to oscillatory motion in the Taylor experiment, Nature (London) 288, 567 (1980).

[44] K. A. Cliffe, J. J. Kobine, and T. Mullin, The role of anomalous modes in Taylor-Couette flow, Proc. R. Soc. London Ser. A 439, 341 (1992).

[45] T. Mullin, M. Heise, and G. Pfister, Onset of cellular motion in Taylor-Couette flow, Phys. Rev. Fluids 2, 081901 (2017).

[46] F. Wendt, Turbulente strömungen zwischen zwei rotierenden konaxialen zylindern, Ing. arch 4, 577 (1933). 